\title{
High-Speed Observations of Sprite Streamers
}

\author{
H. C. Stenbaek-Nielsen • T. Kanmae • M. G. McHarg • \\ R. Haaland
}

Received: 3 November 2012/ Accepted: 14 February 2013/Published online: 15 March 2013

(C) The Author(s) 2013. This article is published with open access at Springerlink.com

\begin{abstract}
Sprites are optical emissions in the mesosphere mainly at altitudes $50-90 \mathrm{~km}$. They are caused by the sudden re-distribution of charge due to lightning in the troposphere which can produce electric fields in the mesosphere in excess of the local breakdown field. The resulting optical displays can be spectacular and this has led to research into the physics and chemistry involved. Imaging at faster than 5,000 frames per second has revealed streamer discharges to be an important and very dynamic part of sprites, and this paper will review high-speed observations of sprite streamers. Streamers are initiated in the 65-85 km altitude range and observed to propagate both down and up at velocities normally in the $10^{6}-5 \times 10^{7} \mathrm{~m} / \mathrm{s}$ range. Sprite streamer heads are small, typically less than a few hundreds of meters, but very bright and appear in images much like stars with signals up to that expected of a magnitude -6 star. Many details of streamer formation have been modeled and successfully compared with observations. Streamers frequently split into multiple sub-streamers. The splitting is very fast. To resolve details will require framing rates higher than the maximum 32,000 fps used so far. Sprite streamers are similar to streamers observed in the laboratory and, although many features appear to obey simple scaling laws, recent work indicates that there are limits to the scaling.
\end{abstract}

Keywords Sprites $\cdot$ Mesosphere $\cdot$ Streamers $\cdot$ Lightning $\cdot$ Electric discharges

\author{
H. C. Stenbaek-Nielsen $(\bowtie) \cdot T$. Kanmae \\ Geophysical Institute, University of Alaska, 903 Koyukuk Drive, Fairbanks, AK, USA \\ e-mail: hnielsen@gi.alaska.edu \\ T. Kanmae \\ e-mail: tkammae@gi.alaska.edu \\ M. G. McHarg \\ The United States Air Force Academy, Colorado Springs, CO, USA \\ e-mail: Matthew.Mcharg@usafa.edu \\ R. Haaland \\ Fort Lewis College, Durango, CO, USA \\ e-mail: haaland_r@fortlewis.edu
}




\section{Introduction}

Sprites are spectacular optical emissions in the mesosphere caused by transient electric fields originating from cloud to ground lightning discharges. They typically occur in the altitude range 55-90 km, although sprites have been observed reaching all the way down to near cloud tops at $\sim 25 \mathrm{~km}$ altitude and up to about $100 \mathrm{~km}$ altitude. They last from a few $\mathrm{ms}$ to more than $100 \mathrm{~ms}$ with many very dynamic features, for example streamers, which are obvious objects for investigations using high-speed imaging.

The sprites are part of a larger set of optical phenomena observed in the mesosphere. Collectively, they are called Transient Luminous Events (TLEs), and a number of these are illustrated in Fig. 1. While sprites (upper right of the figure) originate in the mesosphere in the $65-85 \mathrm{~km}$ altitude range, blue jets and gigantic jets (center lower left) both originate from near the top of the clouds. Recent reviews of TLEs have been given by Neubert et al. (2008) and Pasko (2010) and of high-speed imaging of sprites by Stenbaek-Nielsen and McHarg (2008).

In high-speed images, sprite events typically contain a number of distinct identifiable features: Halo, down, and upward moving streamers, glow, and beads. They result from the quasi-static mesospheric electric field generated by the redistribution of electric charges due to the lightning discharge (Pasko et al. 1997). The individual features may vary considerably in brightness and all may not be detected, nor may they be present, in individual sprite events. At the very start of an event, we may also observe an elve, which is from optical emissions generated by the electromagnetic pulse radiated from the lightning discharge exciting airglow emissions at an altitude near $95 \mathrm{~km}$ (Fernsler and Rowland 1996; Fukunishi et al. 1996; Inan et al. 1997; Veronis et al. 1999; BarringtonLeigh et al. 2001). Because of the different mechanism, it is appropriate not to consider the elve as part of the sprite itself; however, from an analysis point of view, the elve, when it

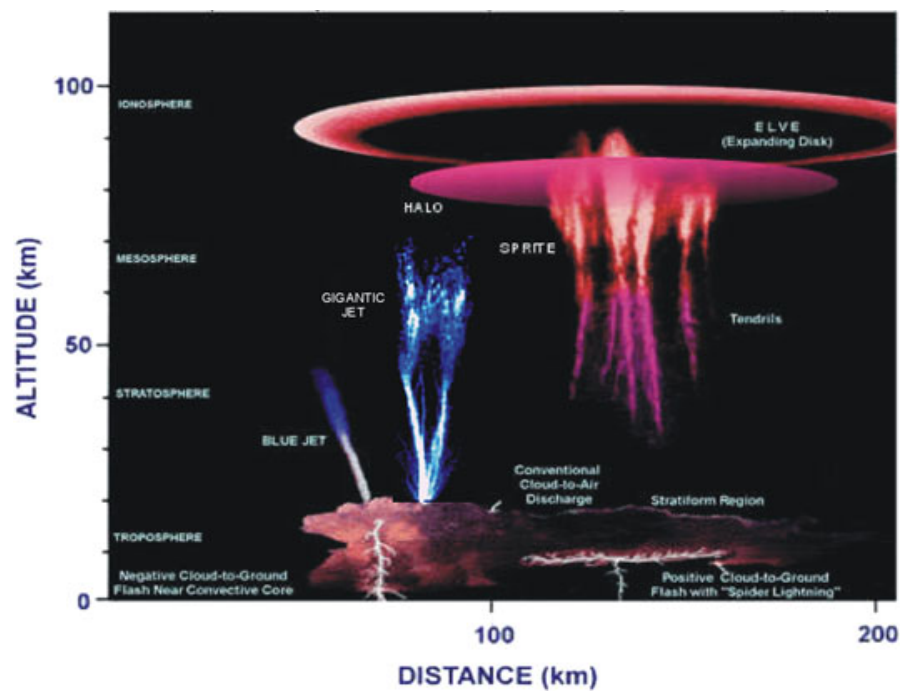

Fig. 1 Schematic of lightning-associated mesospheric optical features. Figure was provided by D. D. Sentman based on artwork originally from Lyons et al. (2000), illustrating features present in video observations. Highspeed observations have revealed that the tendril structures of the sprites (center right) are actually formed by fast moving streamer heads. Figure adapted from Stenbaek-Nielsen and McHarg (2008) 
can be identified, serves as an excellent timing marker for the causal lightning strike, and therefore is often included when describing sprite observations.

The sprite halo is a large, diffuse, pancake-like luminous feature (Barrington-Leigh et al. 2001), while the sprite streamers are the result of an electrical breakdown in the local atmosphere. The onset altitude of the initial sprite streamer is observed to be somewhere in the 65-85 km range (Stenbaek-Nielsen et al. 2010). Liu (2012) has suggested low altitude streamer onset may be related to electron detachment of $\mathrm{O}^{-}$causing ionization in the halo to descend farther down. Streamers occurring immediately after the causal lightning discharge tend to have their onset at higher altitude than delayed sprites (Hu et al. 2007; Li et al. 2008; Gamerota et al. 2011). The onset altitude dependence on delay time is likely related to effects of the lightning-associated continuous current in the troposphere, as first suggested by Cummer and Füllekrug (2001), which adds to the electric field in the mesosphere, and eventually, if conditions are right, can cause streamers to form. Recently, Luque and Gordillo-Vázquez (2012) and Liu (2012) note that electron detachment processes may contribute to the streamer formation and Luque and Gordillo-Vázquez (2012) further developed the idea to formulate a quantitative model for long delayed sprites and their onset altitude.

The sprite glow is from optical emissions in the onset region. It is more or less stationary and is much longer lasting than the streamers. In video images, the glow is often the most prominent sprite feature. In earlier papers (Stenbaek-Nielsen et al. 2007; McHarg et al. 2007; Stenbaek-Nielsen and McHarg 2008), we have speculated that the glow could be associated with chemical processes initiated by streamer activity. This provided the incentive for Sentman et al. (2008) to explore the temporal development of optical emissions from a cascade of chemical processes initiated by streamers. A similar analysis has also been presented by Gordillo-Vázquez (2008, 2010). However, it is now clear that the glow is more likely caused by electric fields associated with negative charge accumulating in the streamer channel (Luque and Ebert 2010; Liu 2010; Li and Cummer 2012; Qin et al. 2012; Kosar et al. 2012).

Finally, beads are small optical features which have received relatively less attention; they move slowly, if at all, and typically last longer than any of the other sprite features (Sentman et al. 1996; Stenbaek-Nielsen et al. 2000; Moudry 2003; Moudry et al. 2003; Gerken and Inan 2002, 2003; Marshall and Inan 2006). They often appear along streamer channels and Luque and Gordillo-Vázquez (2011) have suggested that they are caused by streamers interacting with localized atmospheric electrical charge structures.

The optical emissions in sprites are dominated by the first positive band of molecular nitrogen $\left(\mathrm{N}_{2}\right.$ 1P) emitting in the near infrared (Mende et al. 1995; Hampton et al. 1996; Morrill et al. 1998; Bucsela et al. 2003; Kanmae et al. 2007). Ionization emissions from the $\mathrm{N}_{2}{ }^{+} 1 \mathrm{~N}$ band system have been reported from ground and aircraft photometry by Armstrong et al. (1998), Suszcynsky et al. (1998), Armstrong et al. (2000), and Morrill et al. (2002), and from space by Kuo et al. (2008) and Adachi et al. (2008). Spectra of individual sprite streamers and sprite glow were recorded by Kanmae et al. (2010a, b). No ion emissions were detected, but the streamers had a higher fraction of blue emissions $\left(\mathrm{N}_{2} 2 \mathrm{P}\right)$ than the glow, indicating, as expected, that more energetic processes excite streamer emissions (Liu and Pasko 2004, 2005). Spectral modeling by Gordillo-Vázquez et al. (2012) points out differences between expected spectra from elves, halos, and streamers, but these differences have not yet been verified by observations.

This paper will concentrate on characteristics of sprite streamers as derived from observations using high-frame rate imagers. Streamers are very dynamic features and their temporal development can best be investigated using high-speed imagers. Most of our 
observations have used 10,000-20,000 fps, but we have recently recorded at 32,000 fps. Sprite streamers are relatively small, typically less than a few $100 \mathrm{~m}$ in diameter, which in most sprite recordings is comparable to the image pixel resolution. To better resolve the streamers, we have in several recent observation campaigns used longer, 300-500 mm, focal length lenses which have provided spatial resolution (evaluated at the distance of the sprite) as low as $15 \mathrm{~m}$.

Sprite streamers have been modeled by several groups (Liu and Pasko 2004; Ebert et al. 2006; Chanrion and Neubert 2008; Liu et al. (2009a); and references therein). Modeling has successfully reproduced most sprite features observed, allowing a detailed evaluation of the processes involved. Recently, Luque and Ebert (2009), Qin et al. (2011), Li and Cummer (2012) and Luque and Gordillo-Vázquez (2012) have presented modeling efforts placing streamer formation as part of the larger response of the mesosphere to an electric pulse caused by a cloud-to-ground lightning discharge, and modeling efforts are under way by several groups to relate the lightening pulse shape and charge transport, inferred from electric and magnetic observations, to actual sprite features observed optically with highspeed imagers. Also the potential impact on the global electric circuit is being investigated (Rycroft and Odzimek 2010).

Sprite streamers are phenomenologically similar to electrical discharges observed in laboratory experiments (see reviews by Pasko 2007; Ebert et al. 2010), and it is natural to compare analyses of streamers observed in laboratory experiments with sprite streamer observations. This may indicate the extent to which laboratory experiments, typically performed at ground pressure, can be related to sprite streamers in near vacuum at $80 \mathrm{~km}$ altitude where, although the many processes involved may be the same, their ranking in importance may be different.

\section{The Need for High-Speed Imaging of Sprite Streamers}

Sprite streamers develop very rapidly and their temporal development can only be documented using high-speed imagers. This is illustrated in Fig. 2 where three images of the same sprite event, but with different exposure time, are shown. The recordings were made

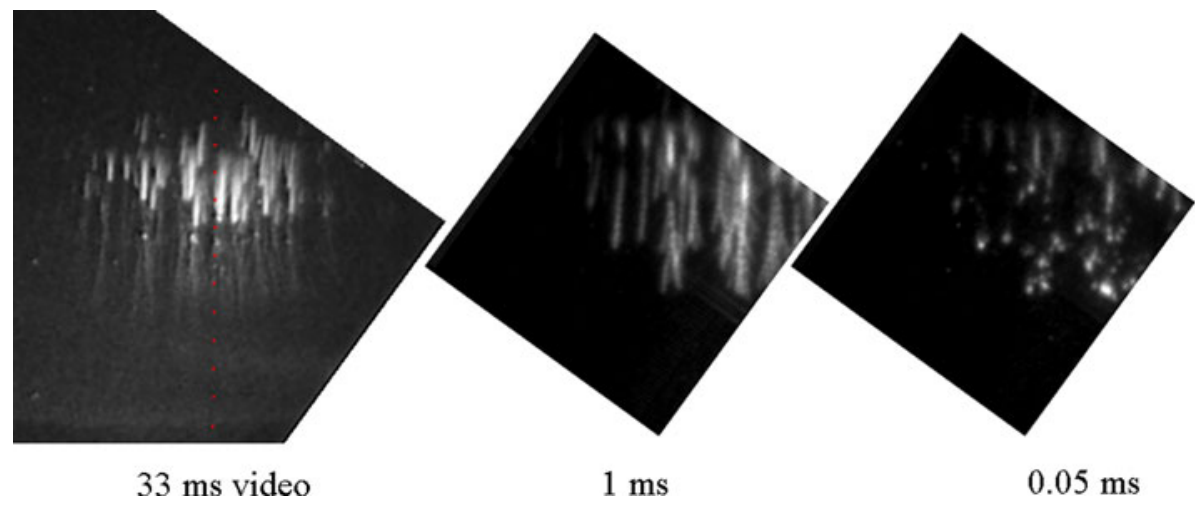

Fig. 2 Sprite observed from Langmuir Laboratory, New Mexico, on July 9, 2005 at 06:05:46 UT. The three frames were recorded at the same time, but with different exposure times, 33,1 , and $0.05 \mathrm{~ms}$, respectively, to illustrate the need for high time resolution in order to resolve sprite streamers. Only at the sub-millisecond exposure is the true nature of the streamers revealed. Figure adapted from Stenbaek-Nielsen and McHarg (2008) 
with the imagers on an equatorial mount, but we have rotated the images so that they are displayed with the horizon across the page. The left image is a $33 \mathrm{~ms}$ (normal US video rate) exposure of the event. In the middle is the event recorded with $1 \mathrm{~ms}$ exposure, and to the right at $50 \mu \mathrm{s}$. The center image, although recorded at 1,000 fps, is not radically different from the video image to the left. Only in the right image do we see the true nature of the streamers: They are actually small optical features, streamer heads, propagating downwards at high speed. Based on video data, the streaks in the two images to the left are called 'tendrils,' and they are simply the result of insufficient time resolution much like in nighttime photographs head or tail lights on cars will show up as long streaks of light.

\section{Sprite Streamer Morphology}

One of the first findings from high-speed sprite imaging was that sprite streamer activity starts with a downward-propagating streamer (Stanley et al. 1999; Cummer et al. 2006; McHarg et al. 2007; Stenbaek-Nielsen and McHarg 2008). Upward propagating streamers, when observed, appear later, from a lower altitude, and from sprite structures presumably formed by the preceding downward-propagating streamers (Stenbaek-Nielsen and McHarg 2008). Figure 3 shows a $4.5 \mathrm{~ms}$ image time series documenting this. The event was recorded at 10,000 fps from Langmuir Laboratory, NM, on July 9, 2005 at 04:15:17 UT. We only have observations from that one station, and the altitude scale on the figure was derived assuming the sprite occurring at the range of the causal lightning strike as reported by the National Lightning Detection Network (NLDN).

The high-speed sprite observations have also provided more detailed information about the differences between C-sprites (columnar sprites) and carrot sprites. The classification of $\mathrm{C}$ and carrot sprites is based on how the sprites appear in video recordings (Wescott et al. 1998). The high-speed images show that the difference is due to the upward propagating streamers (Stenbaek-Nielsen and McHarg 2008). If there are no upward streamers the sprite would be characterized as a $\mathrm{C}$ sprite; if there are many, it would be a carrot sprite. It must be noted that the number of upward streamers relative to downward can vary greatly from event to event, and it is often difficult to decide on the type. We have many examples where the sprite starts as a classical multiple $\mathrm{C}$-sprite, but then, sometime later several of the individual C-sprites will develop upward streamers and the event becomes a combination of $\mathrm{C}$ and carrot sprites.

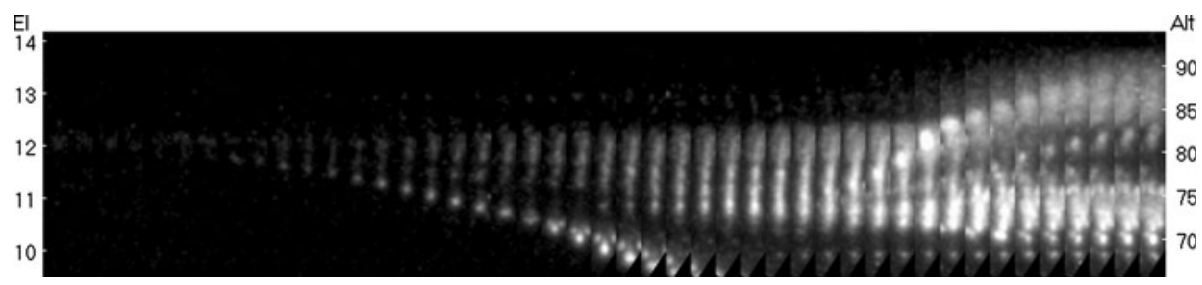

Fig. 3 Langmuir, NM, July 9, 2005 04:15:17 UT, 10,000 fps. Image time series constructed of successive tall narrow image sections cut from the original images. The series covers $4.5 \mathrm{~ms}$ of data. The sprite event starts at an altitude of $82 \mathrm{~km}$ with a downward-propagating streamer. Later, from an altitude of about $73 \mathrm{~km}$ and from existing luminous structure, an upward propagating streamer is seen. The scale on the left is the elevation angle (in degrees) and on the right altitude (in $\mathrm{km}$ ) assuming that the sprite is at the distance of the causal lightning discharge 
The observation that sprites start with a downward streamer was somewhat unexpected as models, for example Liu and Pasko (2004), predicted streamer formation with both a downward (positive) streamer propagating in the direction of the causal electric field, and simultaneously, an upward propagating (negative) streamer, so-called double-headed streamers. The explanation for downward only at onset has been sought in the formation of screening charges (polarization) in the halo or at the bottom of the sharply increasing background electron density profile, where the streamer onset is likely to occur. This polarization will screen the driving electric field above the onset altitude, thus effectively preventing the upward propagating streamer from forming (Qin et al. 2011, 2012; Li and Cummer 2012; Luque and Ebert 2010). Another possibility has been presented by Liu et al. (2012) and Kosar et al. (2012). They have modeled streamer formation from an isolated ionization column under a sub-breakdown electric field and found that only the positive (downward-propagating) streamer will develop.

Recently, Qin et al. (2012) have brought up the issue of double-headed streamers. They find that the sprite streamer development will critically depend on altitude and that doubleheaded streamers are predicted to be present in streamers formed at lower altitude. The model used is based on the highly successful sprite model developed by Pasko and Liu and co-workers over the last decade (Pasko et al. 1997; Liu and Pasko 2004; Liu et al. 2009a, b; Qin et al. 2011). Figure 4 (adapted from Qin et al. (2012), figs. 1, 2) shows model results relevant to the discussion here.

Fig. 4 Top panel shows the reduced electric field and the expected type of streamer at different altitudes. Bottom panel shows an image time series of modeled double-headed streamers with onset at 75 and $77 \mathrm{~km}$ altitude. (Figure provided by J. Qin based on Qin et al. (2012), Figs. 1, 2)
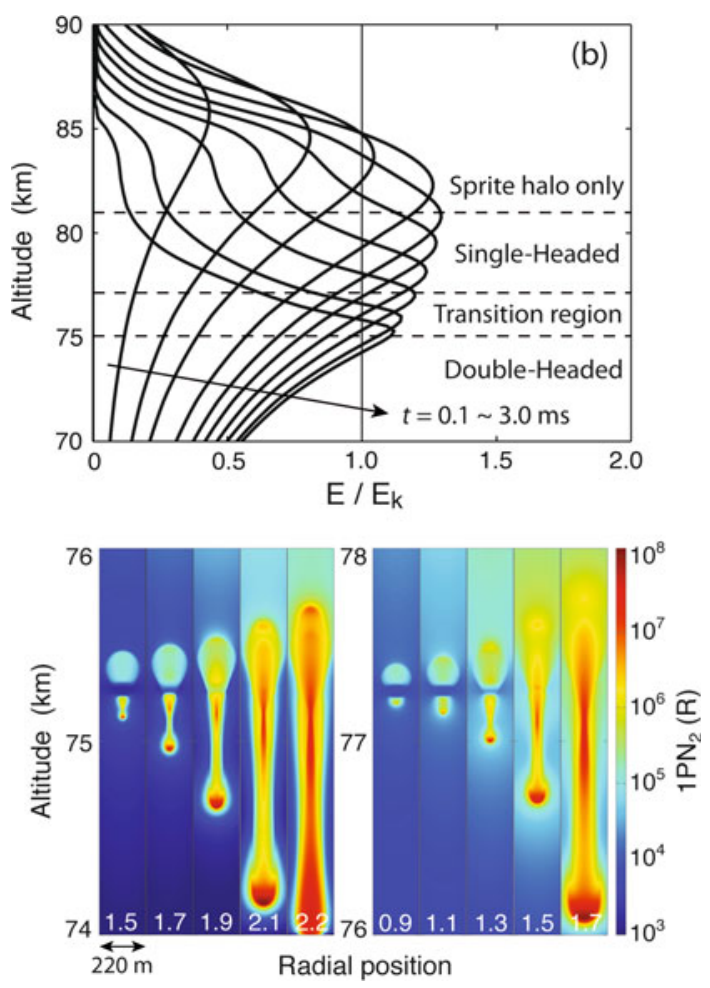
Figure 4, top, illustrates the different altitude regimes. Above $81 \mathrm{~km}$ the model background electron density increases sharply (essentially the bottom of the ionosphere) and streamer development will not occur here; only the halo will be produced. Below comes an altitude region where we should only observe single-headed streamers but, below $\sim 75 \mathrm{~km}$ altitude, streamer formation should result in double-headed streamers, that is, streamers should have both downward and upward propagating branches. Figure 4, bottom, shows the expected streamer luminosity structure in time series at $0.2 \mathrm{~ms}$ interval for streamers with an onset altitude of 75 and $77 \mathrm{~km}$. The upward propagating streamers have a somewhat different appearance and propagate slower than the downward. Qin et al. (2012) point out that the upward streamer may be difficult to see against glow present in the region. For the two model examples shown in Fig. 4, the brightness of the upward streamer is less than the downward streamer, but not so much that the luminosity would never be detected by high-speed imagers. In Fig. 3, we showed the initial streamer formation from an event with onset at an altitude of $81 \mathrm{~km}$. Figure 5, in a similar format, shows the initial streamer formation with onset at an altitude of $65 \mathrm{~km}$. Both examples show the initial streamer as a downward-propagating streamer. This is the case in all our observations, regardless of altitude, where the initial streamer can be identified. Sprite glow is often detected in the onset region. The glow often appears to slowly extend upwards, see for example Fig. 3 above or Fig. 5 in Stenbaek-Nielsen and McHarg (2008) and might be associated with the model upward streamer, but the observed speed appears to be significantly less than the model predicts. However, a direct comparison of the two onset altitudes may be misleading as the total charge moment change from the lightning discharge must be considered as well (J. Qin, personal communication, 2012). As mentioned above, the previous modeling has been overall very successful, and at the time of this writing, details in many of our high-speed sprite events are being compared with the model predictions to try to reconcile model results and observations.

Another model for streamer formation has been proposed by Luque and Ebert (2009). In this model, the streamer is formed from a downward-propagating ionization wave in the halo, and the streamer would appear from near the bottom of the halo as has been observed by Cummer et al. (2006). We do not always observe a halo prior to streamer formation, for example in Fig. 3 there is no optical halo emissions detected, but this could simply be

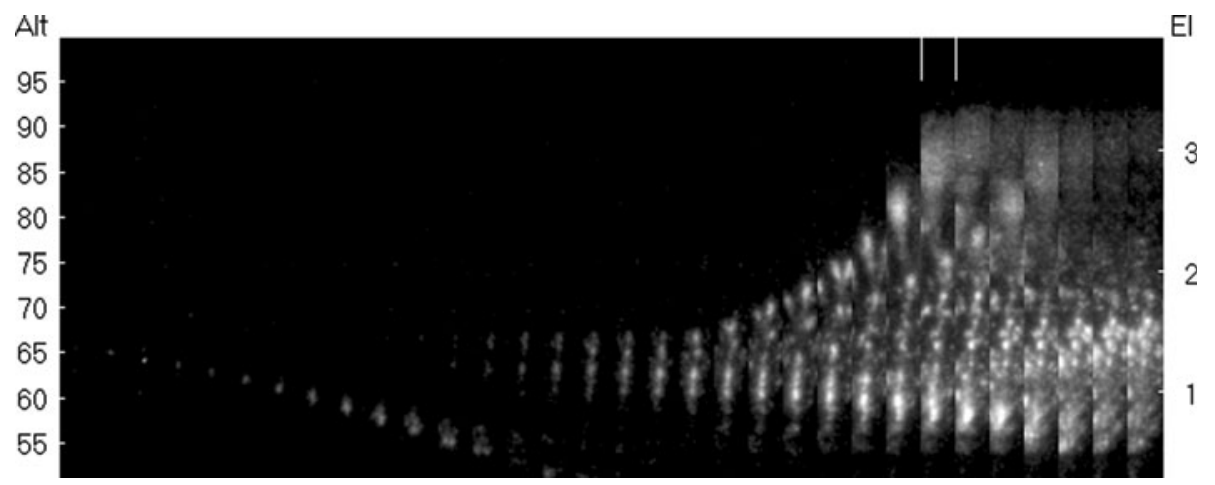

Fig. 5 Image time series of a sprite with 66-km streamer onset altitude. The recording was made at 12,500 fps. The sprite was observed from Langmuir Laboratory on July 3, 2008 at 07:22:04 UT. Only a downward-propagating streamer is observed initially. The format of the figure is the same as used in Fig. 3 


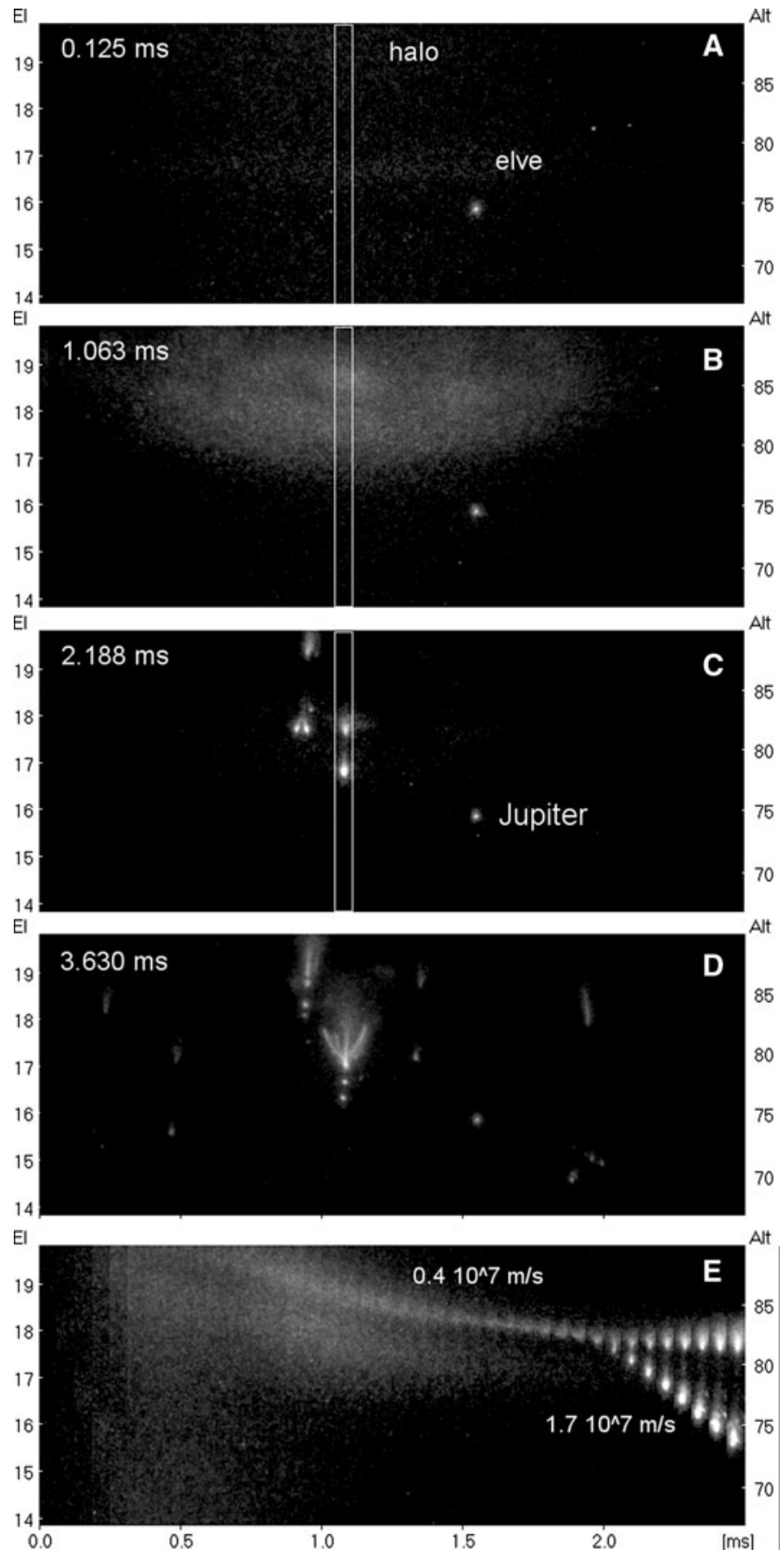


4 Fig. 6 Streamer development in event on August 27, 2009 at 09:15:23 UT. Top image, A, has the elve across the center part of the image, and the halo is just entering the field from above. Image B shows the halo with considerable spatial structure. Image $\mathrm{C}$ shows the early streamers, and D shows the central streamer with glow from upward propagating streamers. This event is an example of an event that is a combination of multiple C-sprites and carrots. Panel E shows a 2.5-ms image time series with the halo streamer development. The time series is formed from the image sections outlines by the white box in images A-C (Image D is after the end of the time series). The streamer is seen to form from structure in the halo descending with the halo and forms after the main halo has faded. El and alt are as defined in the caption to Fig. 3

because the imager is not sensitive enough. In many events, we see the streamer onset only after the main halo emissions have died out. Qin et al. (2011) note that such a delay would argue against a continuous process, but Luque and Gordillo-Vázquez (2012) attribute such delays to chemical effects related to electron detachment processes. In several such examples, there are luminous structures descending with the halo and they remain visible well after the main halo has faded and eventually spawn streamers. A possible interpretation is that they are the optical manifestation of the ionization wave. An example is shown in Fig. 6 with 4 images showing the elve, halo, streamers, and an image time series, similar to Figs. 3 and 5 above, showing the temporal streamer development.

The event in Fig. 6 was observed from the US National Science Foundation HIAPER aircraft flying at $14 \mathrm{~km}$ altitude over Oklahoma on August 27, 2009 at 09:15:23 UT. The recording was made at $16,000 \mathrm{fps}$ with $25 \mu$ s exposures. The images are $640 \times 256$ pixels with 12 bit dynamic range, and the field of view is $15.2^{\circ} \times 6.0^{\circ}$. The top image, A, has the elve horizontally across the center; next image, B, shows the halo with well-defined spatial structure; images $\mathrm{C}$ and $\mathrm{D}$ show the streamers, and finally, $\mathrm{E}$ has the image time series formed of slices from 40 consecutive images, $2.5 \mathrm{~ms}$, starting with the first detection of the sprite elve. The white box on the full images shows the image region extracted for the time series in panel $\mathrm{E}$, and the time relative to the first appearance of the elve is given in the upper left corner. The altitude scale was derived assuming that the sprite was at the distance of the causal lightning strike as recorded by NLDN. The bright bead-like feature at center right is the planet Jupiter. The halo descends at $0.4 \times 10^{7} \mathrm{~m} / \mathrm{s}$. Because of the rapid descent, the optical structure in the halo cannot represent features in the background atmosphere. We note that we have a number of other examples with significantly faster downward motion. While the main halo luminosity fades, the structure in the halo continues and eventually spawns a streamer which descends at $1.7 \times 10^{7} \mathrm{~m} / \mathrm{s}$. The streamer clearly forms after the main halo has faded, and it may be that the structure is actually the ionization wave proposed by Luque and Ebert (2009).

The velocity of sprite streamers is typically in the $10^{6}-10^{8} \mathrm{~m} / \mathrm{s}$ range (Stanley et al. 1999; Moudry et al. 2003; McHarg et al. 2002, 2007; Cummer et al. 2006). Upward streamers are generally slightly faster; the highest velocity we have observed is $1.4 \times 10^{8}$ $\mathrm{m} / \mathrm{s}$ (almost half the velocity of light) (McHarg et al. 2002). As seen in Figs. 3, 5, and 6, the downward streamers accelerate after formation. Eventually, the streamers reach a maximum velocity after which they slow down and eventually fade at lower altitude. McHarg et al. (2007) find acceleration and deceleration values in the $10^{5}-10^{10} \mathrm{~m} / \mathrm{s}^{2}$ range. An analysis covering the entire altitude range of a number of streamers has been done by $\mathrm{Li}$ and Cummer (2009). They find that the deceleration after the peak velocity is reached is nearly constant at $10^{10} \mathrm{~m} / \mathrm{s}^{2}$, and remarkably, the deceleration is nearly the same across most sprites. In a later paper, $\mathrm{Li}$ and Cummer (2012) note that the streamers terminate where the electric field falls below $0.05 E_{\mathrm{k}}$ independent of altitude ( $E_{\mathrm{k}}$ is the local breakdown electric field). $\mathrm{Li}$ and Cummer (2009) also find that in a sprite event with 
multiple streamers, the streamers at the center of the event propagate faster and terminate at a lower altitude than streamers toward the edge. They speculate that this may reflect a lower electric field near the edges of an event. We find in our data similar characteristics, particularly in most multiple C-sprite events. Luque and Ebert (2010) have modeled streamer propagation in an atmosphere with a varying density and in a constant electric field. Their model shows the streamer to accelerate to a maximum velocity after which it decelerates at a nearly constant value in agreement with Li and Cummer (2009). However, $\mathrm{Li}$ and Cummer (2012) note that the details of this simulation are at variance with their observations, and they suggest that a constant electric field, as used by Luque and Ebert (2010), may not represent the real situation.

In contrast to the downward-propagating streamers, which gradually fade into their low altitude termination, the upward propagating streamers typically stop fairly suddenly at the upper altitude limit with a pronounced puff of light. See, for example, Figs. 10 and 12 in Stenbaek-Nielsen and McHarg (2008) or Fig. 3 in Stenbaek-Nielsen et al. (2000). This may be related to the altitude where the electric conductivity becomes too large to support propagation (Pasko and Stenbaek-Nielsen 2002).

Streamers are relatively small, typically less than a few $100 \mathrm{~m}$ in diameter. Gerken et al. (2000) and Marshall and Inan (2006) have made sprite observations using a telescope and report streamer widths as small as a few tens of meters. Most of our high-speed observations have a pixel resolution of 100-200 m effectively preventing reliable scale sizes to be measured. In these data, the streamers appear in the images very similar to stars. In several recent observation campaigns, we have used longer focal length lenses to better resolve spatial structures in sprites (McHarg et al. 2007; Kanmae et al. 2012). Evaluated at the distance of the sprite streamers, the pixel resolution has been as low as $15 \mathrm{~m}$. Some of these data will be discussed in Sects. 5-7 below.

Positive streamers propagate in the direction of the ambient electric field and negative streamers, in the opposite direction. The positive streamers will carry positive charge downward leaving the onset region negatively charged (Liu 2010; Luque and Ebert 2010). The field from this charge is responsible for the glow observed there, and if the field strength increases to above the local breakdown field, $E_{\mathrm{k}}$, negative streamers propagating upwards will be formed. This model concept, developed by several groups ( $\mathrm{Li}$ and Cummer 2011; Luque and Ebert 2010; Qin et al. 2011), naturally explains that the upward streamers will appear later than the initial downward streamer, and it also explains the larger horizontal velocity component observed in the upward streamers creating the broad tops of carrot sprites. If there is sufficient conductivity between the onset region and the ionosphere, the field will not reach breakdown and no negative streamers will be formed. It follows that we should then expect $\mathrm{C}$ type sprites (no upward streamers) to have their onset at relatively high altitude, and our observations appear to support this (Stenbaek-Nielsen et al. 2010).

Streamers propagating through the neutral atmosphere will create a high-conductivity negatively-charged channel (Luque and Ebert 2010; Gordillo-Vázquez and Luque 2010; Liu 2010; Kosar et al. 2012). It is significant to note that Gordillo-Vázquez and Luque (2010) find that the high-conductivity channel will last for several minutes, which is well past any sprite-associated optical emissions. This provides a good explanation for the 'reignition' of sprite structures by a later sprite reported by Stenbaek-Nielsen et al. (2000). The streamer channels can attract subsequent positive streamers, and high-speed sprite recordings have shown this to occur (Cummer et al. 2006; Stenbaek-Nielsen and McHarg 2008; Montanyà et al. 2010). Such connection of streamers to earlier-established channels apparently occurs more frequently in sprites than in laboratory discharges (Nijdam et al. 2009). Figure 7 shows a recent example observed on July 11, 2011 at 06:09:57 UT and 
Streamer at contact

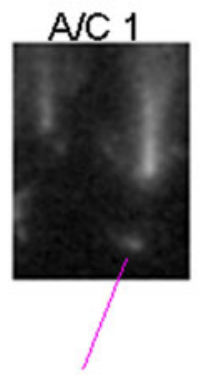

Connecting streamer

AVC 1
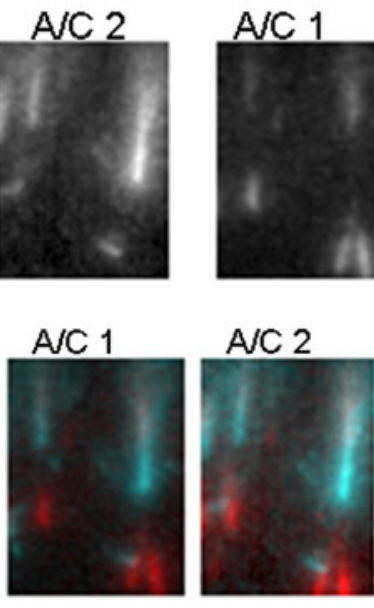

Streamer head
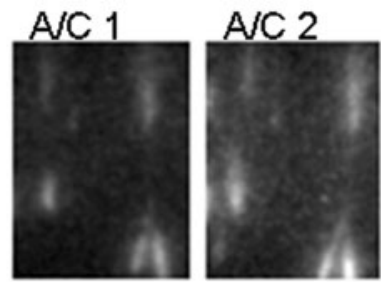

$80.1 \mathrm{~km}$

$71.3 \mathrm{~km}$

\section{Combined image}

Fig. 7 Image segments showing a downward-propagating streamer moving toward and connecting with a previously established streamer channel. The event was observed from two aircraft over Iowa on July 11, 2011 at 06:09:57 UT and recorded at 10,000 fps with 0.1 ms exposures. The upper left pair of images from the two aircraft, A/C 1 and A/C 2, shows the streamer (position annotated in upper left image) at the time of connection with the channel, which was formed $1.4 \mathrm{~ms}$ earlier by a downward-propagating streamer head as shown in the image pair upper right. At the bottom is a combined image with the upper left images in blue and the upper right in red. The triangulated altitude scale is shown to the right. (Data from a sprite aircraft mission sponsored by the Japanese Broadcasting Corporation, NHK)

recorded at 10,000 fps from two aircraft flying $30 \mathrm{~km}$ apart over Iowa in the Mid-West of the United States and at a distance of about $300 \mathrm{~km}$ from the sprite. With observations from two aircraft, the event can be triangulated eliminating the possibility when using one site only, that the streamer did not actually connect with another channel, but only happened to be along the same line of sight.

Figure 7 shows sections from 10,000 fps high-speed images. At top left, we show images from the two aircraft at the time the streamer connects with the previously established channel. The downward streamer was first observed near the upper left corner of the images and propagated first almost straight down then curved over toward the channel and connected near the lower right corner. At the time it connected, it brightened and luminosity was observed moving back up along the streamer track. The formation of the earlier streamer channel is shown top right. The images are from the time the streamer head passes though the point of connection $1.4 \mathrm{~ms}$ earlier. The streamer had just split into two in the previous frame. At the bottom, we show the two sets of images overlaid with the prior streamer in red and the connecting streamer in blue. Triangulation of the event shows the streamer connecting with the channel at $\sim 90^{\circ}$, as one would expect when the channel is highly conducting. It is interesting to note that the streamer did not connect with the channel where the streamer split, but just below. As has been pointed out by Luque and Ebert (2010), connection to other streamer channels appears to be observed in downwardpropagating streamers only. Connections between streamers are not only present in sprites, 
but also in laboratory discharges, and Nijdam et al. (2009) report similar connections at near $90^{\circ}$ indicating electrostatic processes.

\section{Sprite Streamer Brightness}

The sprite streamers are very bright, and it took researchers a number of years to fully appreciate this. The first sprite image was published by Franz et al. (1990). They made the recording during a test of a camera intended for auroral research and identified the optical event as associated with a distant thunderstorm, but they did not appreciate the true brightness and altitude of the optical structure recorded. The true altitude was determined a few years later using observations from two aircraft so the altitude could be triangulated. The results from that mission firmly established sprites as mesospheric events (Sentman et al. 1995).

It is interesting to follow how the reported brightness has increased with our understanding of the spatial and temporal characteristics of sprites. The brightness is an important parameter to derive from observations as this provides the level of physical and chemical activity involved. The first attempt to estimate their brightness was done by Sentman and Wescott (1993) based on events recorded with an intensified video all-sky camera primarily used for auroral observations. The estimated value was '10-50 kR, roughly that of bright aurorae.' (The brightness unit Rayleigh is a photon-based unit commonly used in aeronomy for brightness of optically thin extended sources; $1 \mathrm{R}$ is a photon emission rate of $10^{6}$ photons per second per $\mathrm{cm}^{2}$-column. A short derivation of the unit is given by Chamberlain (1995)). Realizing that sprites vary in brightness on timescales much shorter than the $30 \mathrm{fps}$ recording rate, Sentman et al. (1995) increased the estimate to $600 \mathrm{kR}$.

It soon became clear that higher time resolution than standard video equipment would be needed, and the first high-speed recordings, up to 4,000 fps, were made by Stanley et al. (1999) revealing many new features, but they did not estimate the brightness. Using a newly constructed intensified camera (also originally intended for auroral type research) recording at 1,000 fps, Stenbaek-Nielsen et al. (2000) found that the brightness of the sprites was typically substantially above the $3 \mathrm{MR}$ saturation level for the imager, and they now estimated the maximum brightness to be $\sim 12 \mathrm{MR}$. The observation that sprites will saturate the imagers has also been noted by Gerken et al. (2000), who reported imager saturation at $3 \mathrm{MR}$, and by Marshall and Inan (2005), who used a $1000 \mathrm{fps}$ imager which saturated at $1.2 \mathrm{MR}$.

At 10,000 fps the sprites still saturate the imager, but now an additional new aspect was revealed: Tendrils and branches in the earlier images are actually formed by bright, spatially small, but fast moving streamer heads (McHarg et al. 2007), as shown in Fig. 2 above. Thus, the earlier brightness estimates based on lower time resolution images would have greatly under estimated their brightness. The streamer heads appear in the 10,000 fps images very much like stars do with Gaussian-shaped brightness profiles, as illustrated in Fig. 8 (reproduced from Stenbaek-Nielsen et al. 2007). Where the maximum brightness saturates the detector, the wings of the profile can be fitted as a Gaussian. Absolute calibration of the images was done analyzing star fields using published star magnitudes and spectral characteristics.

The pixel resolution of the event shown in Fig. 8 is about $150 \mathrm{~m}$, but telescopic sprite observations by Gerken et al. (2000) and Marshall and Inan (2006) indicate that the diameter of many streamers can be as low as a few tens of meters. Thus, the streamer heads 


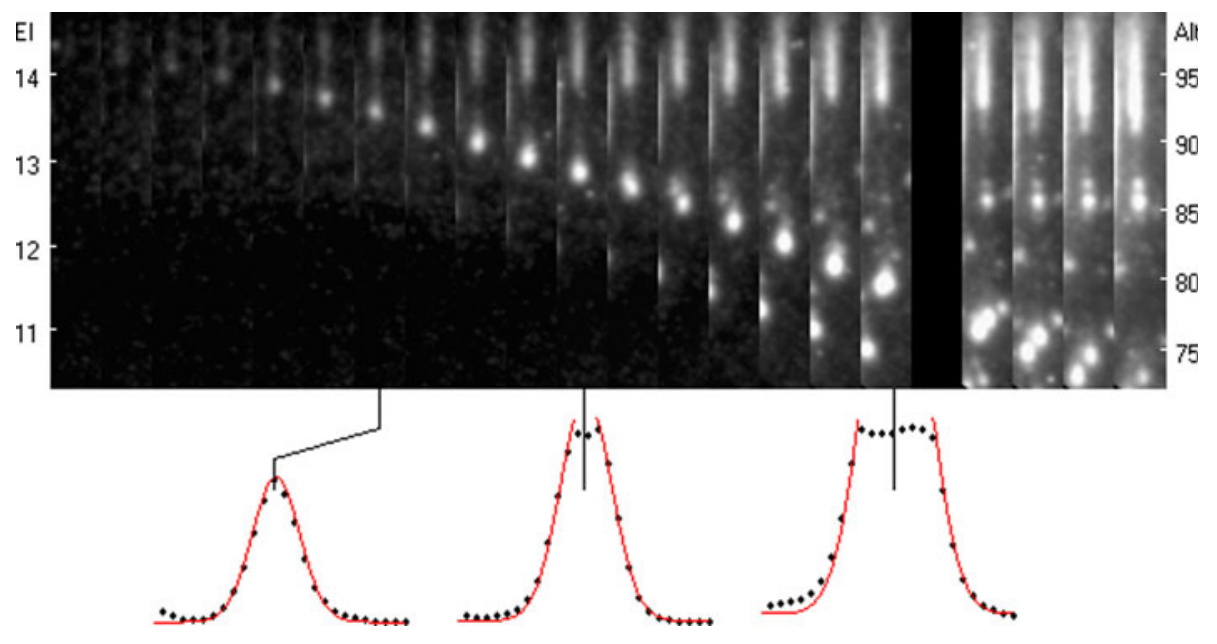

Fig. 8 Strips extracted from successive images showing the downward motion and brightening of a streamer head. Images were recorded at 10,000 fps on July 9, 2005, at 04:38:00 UT. Bottom shows Gaussian profiles fitted to a row across the streamer head center at 3 different times. The dots are the row pixel values. In the left profile, the streamer head does not saturate; just saturates in the middle profile; and in the right profile, the peak brightness is estimated to be 2.2 times the saturation level. In the right profile, the background is higher as luminosity from a nearby structure extends into the left wing of the streamer head. A black strip is inserted where a frame was dropped by the camera. (Figure adapted from Stenbaek-Nielsen et al. 2007)

are mostly unresolved point sources, and a brightness estimate must take this into account. Stenbaek-Nielsen et al. (2007) found, based on a comparison with stars recorded with the same imager and for which we have brightness spectra, that the emission rate for an individual streamer head could be up to $10^{24}$ photons/s. Note, this would be the emissions from the entire streamer head volume. In the high-speed images, $10^{24}$ photons/s would be roughly equivalent to that expected from a star with visual magnitude -6 . The implication is that streamer heads might actually be observable in daylight (Venus which can be seen, although not easily, in daylight has a maximum brightness of about magnitude -4). Assuming a small streamer of only $25 \mathrm{~m}$, as observed by Gerken et al. (2000) and Marshall and Inan (2006), the streamer head brightness would be $100 \mathrm{GR}$, but this is almost certainly an overestimate since the streamers in our high-speed images are likely to be significantly larger in size.

Perhaps more impressive than the brightness numbers given above is the sprite image shown in Fig. 6, panels $\mathrm{C}$ and $\mathrm{D}$, above. The sprite is not particular large nor bright, but the image has the planet Jupiter in the field of view, and the sprite streamers are clearly significantly brighter than Jupiter. The event was recorded at $16,000 \mathrm{fps}$, and we also recorded optical spectra of the sprite at 10,000 fps (Kanmae et al. 2010a).

A comparison of the observed streamer development shown in Fig. 8 with results from a computer simulation has been done by Liu et al. (2009a). The computer simulation model used is that of Liu and Pasko (2004). Figure 9, reproduced from Liu et al. (2009a), shows the streamer development in a format similar to that used in Fig. 8. The modeled streamer emissions are from the first positive band of molecular nitrogen, which dominate the optical emissions in sprites. Because of computer resource limitations, the simulation could only cover the initial $0.3 \mathrm{~ms}$ and not the full $2.2 \mathrm{~ms}$ of the observations shown in Fig. 8. 

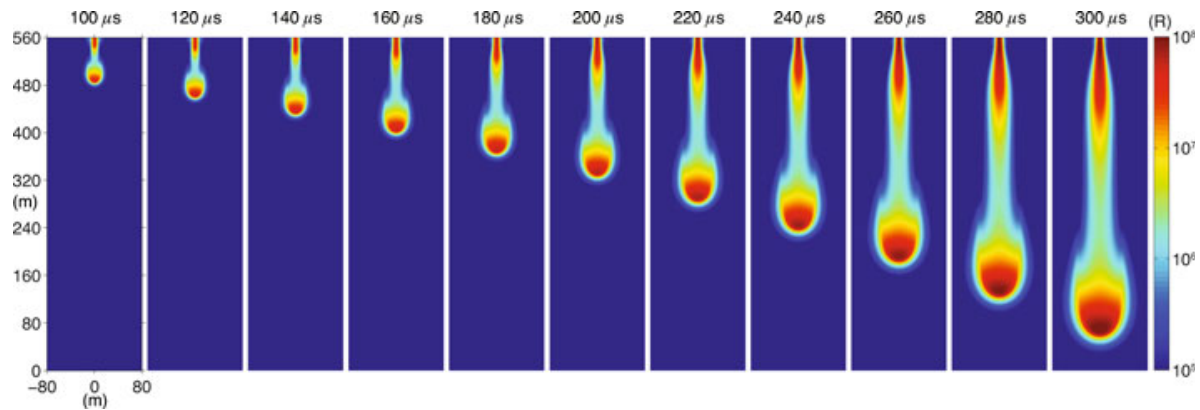

Fig. 9 Model streamer development at $20-\mu$ s intervals starting at $100 \mu$ s and ending at $300 \mu$ s. The streamer is propagating down starting from an altitude of $75 \mathrm{~km}$. The optical emissions are from the first positive band of molecular nitrogen $\left(\mathrm{N}_{2} 1 \mathrm{P}\right)$, and the brightness scale is in Rayleighs. The image format is consistent with that of Fig. 8. (Figure reproduced from Liu et al. 2009a)

However, the essence of the modeled streamer development is clearly similar to that the observed. The streamer head propagates down with increasing speed, size, and brightness. Also, the formation of the bright glow in the upper region of the streamer channel is well simulated.

The brightness of the observed streamer (Fig. 8 above) has been derived from star observations and thus can be compared with the simulations. The observations were made at 10,000 fps with $50 \mu$ s exposures. To facilitate a simple and direct comparison, the simulation was run with $50 \mu$ s integration of the optical emissions (which would correspond to 20,000 fps), and the total streamer head brightness was derived and converted to expected counts in the high-speed images using the imager calibration derived from star fields (Stenbaek-Nielsen et al. 2007). A summary plot of this comparison was given by Liu et al. (2009a) and is adapted here in Fig. 10.

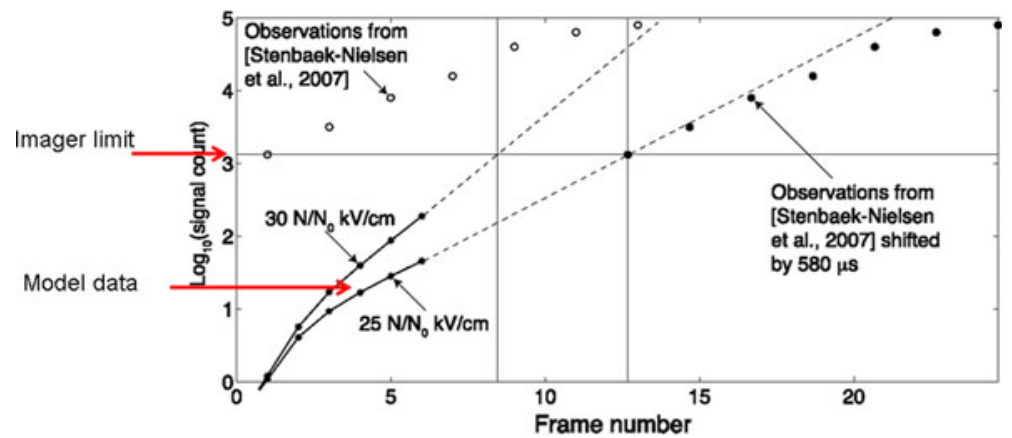

Fig. 10 Signal counts as a function of frame number for the simulation in Fig. 9 and observations in Fig. 8. Circles are the first seven data points presented in Stenbaek-Nielsen et al. (2007, Fig. 3), which correspond to recordings made at $10,000 \mathrm{fps}$ with $50-\mathrm{ms}$ exposure time. The signal counts from the two modeling cases are calculated as if the model streamers are recorded at 20,000 fps with 50-ms exposure time. The simulations stop at frame 6 when stable streamer characteristics (i.e., acceleration, expansion, etc.) are established, and the dashed lines are extrapolations of the simulation brightness for two values of the electric field $\left(30 \mathrm{~N} / \mathrm{N}_{0} \mathrm{kV} / \mathrm{cm}\right.$ and $\left.25 \mathrm{~N} / \mathrm{N}_{0} \mathrm{kV} / \mathrm{cm}\right)$. The slope of the observed streamer counts indicates better agreement with the simulation for the $25 \mathrm{~N} / \mathrm{N}_{0} \mathrm{kV} / \mathrm{cm}$ field. Based on the extrapolated model count, the streamer is first observed $580 \mu$ s after streamer initiation. Figure adapted from Liu et al. (2009a) 
The lower left of Fig. 10 has the model streamer head brightness. Data from two simulations with different background electric fields are shown. Both simulations were done with atmospheric densities corresponding to an altitude of $75 \mathrm{~km}$. The upper trace of the two is for an electric field at the local breakdown electric field value, $E_{\mathrm{k}}=30 \mathrm{~N} /$ $\mathrm{N}_{0} \mathrm{kV} / \mathrm{cm}$, and the other for a lower electric field, $25 \mathrm{~N} / \mathrm{N}_{0} \mathrm{kV} / \mathrm{cm} .30 \mathrm{kV} / \mathrm{cm}$ is the approximate breakdown value at the surface (Raizer 1991, p. 135; Bazelyan and Raizer 1998 , p. 9) and $\mathrm{N} / \mathrm{N}_{0}$ is density scaling to high altitude. The streamer brightness is seen to first increase rapidly, but soon stabilizes to exponential growth, as indicated by the straight line in the logarithmic plot. The growth is then expected to continue at the same rate beyond the end of the simulations assuming no splitting. The simulation results show that the rate of increase in brightness is directly related to the driving electric field, suggesting that observations of the increase in streamer brightness, a parameter which in principle is relatively easy to measure, can provide the driving electric field.

The top of the plot has the observed brightness. The data points shown are the streamer head brightness derived from the initial 7 image sections shown in Fig. 8. Each data point represents the total observed image pixel count across the streamer head as described in Stenbaek-Nielsen et al. (2007). The first data point is from when the streamer is first detected, that is, from when the streamer brightness first appears above the image background, and we have defined this as our effective detection limit as indicated on the figure. The observations are about a factor of 100 brighter than the simulations and therefore cannot represent the initial streamer formation covered by the simulation.

The rate of increase in the observed streamer brightness is seen to be consistent with the simulation results for $25 \mathrm{~N} / \mathrm{N}_{0} \mathrm{kV} / \mathrm{cm}$. Since the slope of the streamer brightness log-plot appears to remain constant at the end of the time covered by the simulation, it is natural to fit the observed streamer brightness points with the extrapolated model brightness curve. Thus, the first observed data point would be $580 \mu$ s after initial streamer formation (the start of the model run), as shown in the upper right of the plot. The analysis shows an overall good agreement between model and observations. A comparison of the model data with spectro-photometric data from the ISUAL instrument in space shows similarly that the driving electric field is close to the local breakdown field (Liu et al. 2009b).

\section{Splitting of Streamers}

High-speed observations of sprite streamers show them frequently to split into several smaller streamers (Stanley et al. 1999; Cummer et al. 2006; Stenbaek-Nielsen and McHarg 2008; McHarg et al. 2010). Splitting is also observed in laboratory experiments (Nijdam et al. 2008), and it appears from modeling to be a characteristic of streamers (Arrayas et al. 2002; Liu and Pasko 2004; Ebert et al. 2006; Luque and Ebert 2011). Figure 11 shows a good example of streamer splitting recorded with high spatial resolution at the Langmuir Observatory, NM, on July 15, 2010 at 07:06:09 UT. The imager had a 500-mm-focal length lens giving a $1.3^{\circ} \times 0.6^{\circ}$ field of view and a pixel resolution of $17 \mathrm{~m}$, significantly better than the 100-200 $\mathrm{m}$ in most of our observations using 50-or 85-mm-focal length lenses. The event was recorded at $16,000 \mathrm{fps}$, with $20 \mu$ s exposures, and two successive frames are shown. The images have been color coded to enhance details. The streamer center left splits into at least 8 smaller streamers between one frame and the next. Thus, the process takes place in less than $62.5 \mu \mathrm{s}$.

A more detailed analysis of streamer splitting has been given by McHarg et al. (2010) using data acquired at 10,000 fps and $50 \mu$ s exposures. The observations were made using 
Fig. 11 Two successive frames recorded at 16,000 fps showing streamer splitting. The field of view is $1.3^{\circ} \times 0.6^{\circ}$. The images have been color coded to enhance details. The streamer at center left splits into at least 8 sub-streamers within $62.5 \mu$ s. The images were recorded from Langmuir Laboratory, NM, on July 15, 2010 at 07:06:09 UT

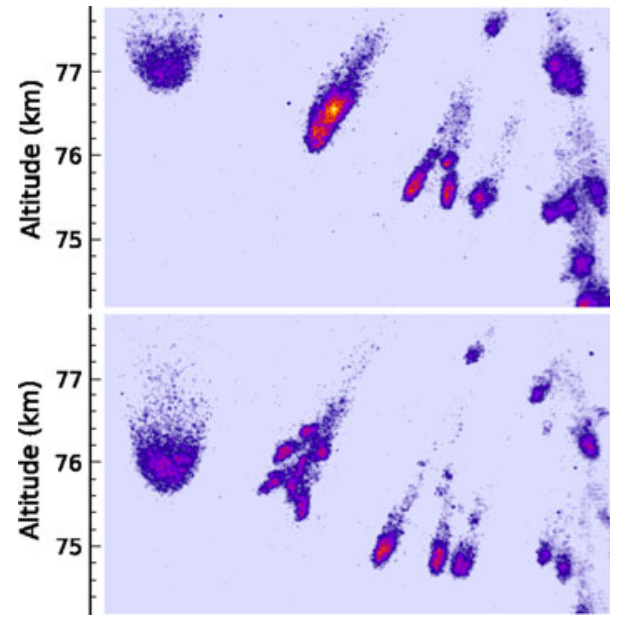

a 300 -mm lens. The field of view was $2.1^{\circ} \times 1.6^{\circ}$, and estimated pixel resolution was $32 \mathrm{~m}$ for the events analyzed. Splitting events were frequently observed in both downward and upward propagating streamers. Figure 12 shows a splitting example from this analysis. The splitting takes place in a downward-propagating streamer (downward streamers are easier to analyze as they typically appear against a dark background in contrast to upward propagating streamers). The streamer is both brightening and increasing in width before it splits, and this was found to be typical. The features in the lower half of the images are all streamers, while the exclamation mark-like feature in the upper left is the sprite glow and a bead. The splitting streamers are, on average, four times brighter than non-splitting streamers, and the median width for non-splitting streamers is $193 \mathrm{~m}$, but $389 \mathrm{~m}$ for splitting streamers indicating that there is a width below which splitting does not occur. Overall, the streamers appear to split into smaller and smaller streamers. The sub-streamers propagate away from the splitting point in a cone, and McHarg et al. (2010) find this cone to be narrower than that found by Nijdam et al. (2008) for splittings in laboratory streamers, but this may be an artifact of the field geometry (Nijdam et al. 2009). We have many recordings with splitting into anywhere from 2 to about 10 sub-streamers, and the examples shown in Figs. 11 and 12 are not at all unusual.

Liu and Pasko (2004) and Ebert et al. (2006) have used their models to investigate the stability of the streamers. Both studies find that, as the streamers grow, the front flattens. This will affect the electric field configuration around the streamer tip, and the streamer geometry becomes unstable. Since both groups use cylindrical symmetry in their streamer modeling, they can only follow the streamer to the initial stages of the instability, but they speculate that the instability will grow into splitting of the streamer. Liu and Pasko (2004) found that their model streamers at an altitude of $70 \mathrm{~km}$ are stable against splitting up to a streamer radius of $97 \mathrm{~m}$. This value agrees well with the median width of $193 \mathrm{~m}$ for nonsplitting streamers observed by McHarg et al. (2010).

The size limit for stability against splitting may be a clue to why sprite streamers often split into many sub-streamers, but laboratory streamers into 2 and only very rarely into 3 (U. Ebert, private communication, 2008; Gordillo-Vázquez 2010). Comparing the densityscaled widths of sprite and laboratory streamers, Kanmae et al. (2012) found that sprite streamers generally are larger than laboratory streamers. The analysis by Kanmae et al. (2012) will be presented in more details in Sect. 7 below. Linking the number of sub- 


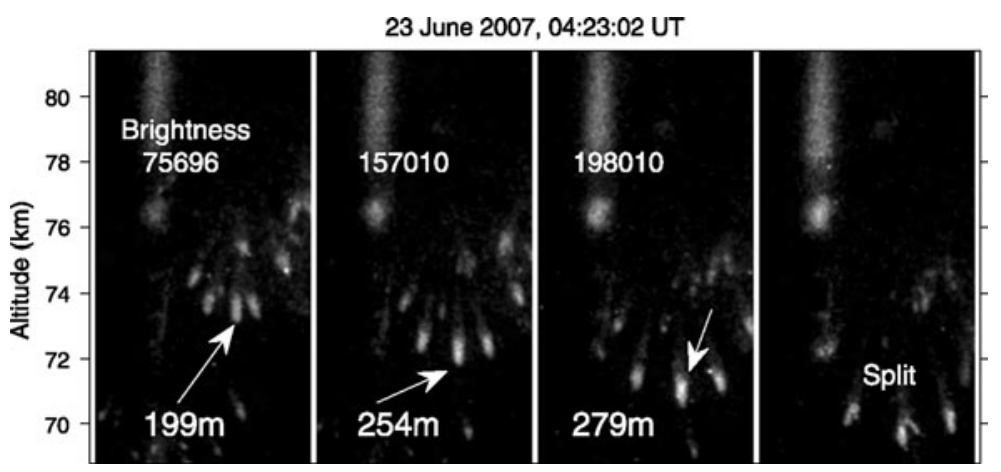

Fig. 12 Four successive images showing splitting of a streamer. The measured FWHM width is given together with a relative brightness value for the streamer pointed to by the arrow. The sprite was observed from the Langmuir Laboratory, NM, on June 23, 2007 at 04:23:02 UT and recorded at 10,000 fps, that is, $100 \mathrm{~ms}$ between images. The altitude was determined using the location of the causative positive cloud to ground stroke determined by NLDN. The streamer head is seen to both grow in width and brighten prior to splitting. Figure reproduced from McHarg et al. (2010)

streamers formed in a splitting to the streamer cross section might provide a clue to why the larger sprite streamers split into more sub-streamers than the smaller laboratory streamers. For example, a 300-m-radius streamer, such as the left streamer in Fig. 11, would have a cross section about 10 times the $97 \mathrm{~m}$ radius stability limit cross section, while laboratory streamers are close to the limiting cross section. We should note that the left streamer in Fig. 11 did split into many sub-streamers at an altitude of $70 \mathrm{~km}$. This was observed in images from a second, larger field of view imager bore sighted with the imager producing the images shown in Fig. 11.

Figures 11 and 12 show the splitting to be a very fast process, and, if the instability is closely linked to an upper limit for stability, then there should be few streamers larger than the stability limit. There may be a finite time needed for the instability to setup during which time larger streamers could exist. Our high-speed observations have many streamers larger than this size limit lasting for highly varying durations, indicating that size alone does not appear to trigger the instability. Briels et al. (2008) analyzing laboratory streamers in the pressure range $0.065-1$ bar measured the ratio of distance travelled between branching to streamer diameter. For thin streamers, they find a ratio of $11 \pm 4$ and a somewhat lower ratio, $8 \pm 4$, for thicker streamers. Therefore, at constant pressure, 'thicker streamers are longer from one splitting to the next, but measured on the scale of their diameter, they branch slightly more frequently' (Briels et al. 2008). A similar analysis has not been done for sprites. For the event shown in Fig. 13, the large streamer to the left travels more than $11 \mathrm{~km}$ before it splits. In contrast, the streamer near the center splits several times with about $4.5 \mathrm{~km}$ between splitting events (Kanmae et al. 2012). Estimating the diameter of the two streamers to be 600 and $250 \mathrm{~m}$, respectively, would indicate larger ratios in sprites. Luque and Ebert (2011) suggest that electron density fluctuations in the front of the streamer head affect the onset of branching, and their analysis suggests that the average length between branching should indeed be longer in sprites than in laboratory streamers at ground pressure. Macroscopic electron density structures, like those proposed by Luque and Gordillo-Vázquez (2011) leading to bead formation, might be involved, although it should be noted that we do not generally observe beads formation where splitting occurs. 
Fig. 13 Optical emissions from a streamer simulation by Luque and Ebert (2010). The optical emissions are from the first positive band of molecular nitrogen. The emissions are integrated in space along a line of sight perpendicular to the streamer propagation and in time over $50 \mu \mathrm{s}$. Figure reproduced from Luque and Ebert (2010)

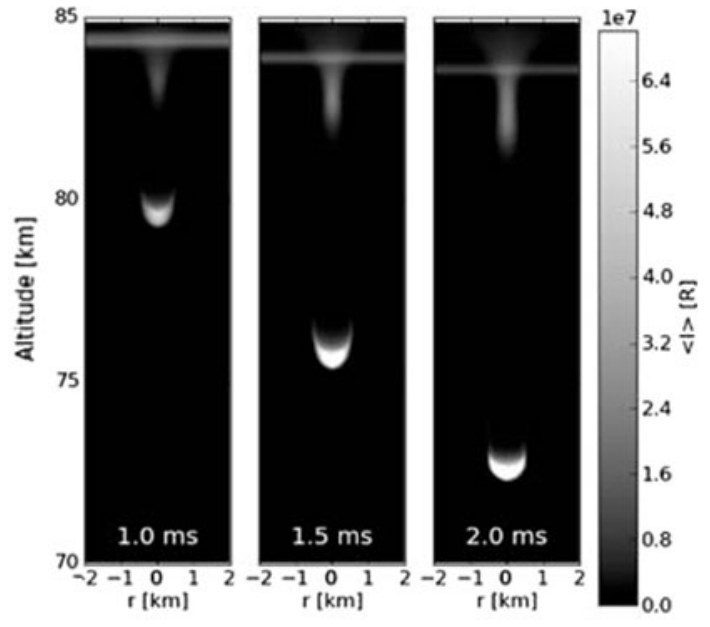

\section{Sprite Streamer Head Shape}

The streamer observations presented in Fig. 11 above have sufficient detail to allow an evaluation of their physical shape. The images were recorded at $16,000 \mathrm{fps}(62.5 \mu \mathrm{s}$ between frames) and $20 \mu$ s exposure. In particular, the left-most streamer is well suited for such an analysis. The downward velocity of the streamer is $2 \times 10^{7} \mathrm{~m} / \mathrm{s}$. In $20 \mu \mathrm{s}$, at a velocity of $2 \times 10^{7} \mathrm{~m} / \mathrm{s}$, the streamer will move $400 \mathrm{~m}$ causing smearing of the streamer in the image. The apparent length of the streamer in the images is roughly $600 \mathrm{~m}$, so the actual length is closer to $200 \mathrm{~m}$. Thus, the shape of the streamer head is more 'saucer'-like with a width of $600 \mathrm{~m}$ and a thickness of $200 \mathrm{~m}$. This streamer is unusually large, and a similar analysis by McHarg et al. (2010) on the larger data set described above in connection with Fig. 12 finds generally smaller widths with a thickness near the pixel resolution of $30 \mathrm{~m}$.

Computer modeling of streamers shows similar 'saucer'-shaped streamer heads. An example with a similar-sized streamer head is shown in Fig. 13 reproduced from Luque and Ebert (2010). This simulation used their 'ionization wave' model for streamer formation (Luque and Ebert 2009) with a 200-m-large initial seed. The emissions are integrated over $50 \mu \mathrm{s}$ and the downward velocity is about $7 \times 10^{6} \mathrm{~m} / \mathrm{s}$. With a similar correction for the smearing effect, the size becomes $500 \times 200 \mathrm{~m}$, about the same as the estimate for the observed streamer in Fig. 11. Other streamer simulations have generally significantly smaller streamers, but the shape of the optical emissions from the streamer head is the same.

\section{Streamers in Sprites and in Laboratory Discharges}

The similarity between streamers observed in the laboratory and in sprites has been noted by many researchers (see reviews by Pasko 2007 and Ebert et al. 2010). The fundamental 
molecular and atomic collision processes involve the same gases and, therefore, one should expect many aspects of the streamer development to scale with atmospheric density (Pasko 2007; Ebert et al. 2010). Figure 14 shows the leftmost streamer in Fig. 13 together with a streamer recorded in a laboratory experiment by Nudnova and Starikovskii (2008). The similarity is quite striking.

The validity of streamer scaling laws is based on an assumption of similar processes, but the relative importance of the many processes involved varies greatly with density/altitude. For example, 3-body processes are relatively unimportant at sprite altitude, but gain rapidly

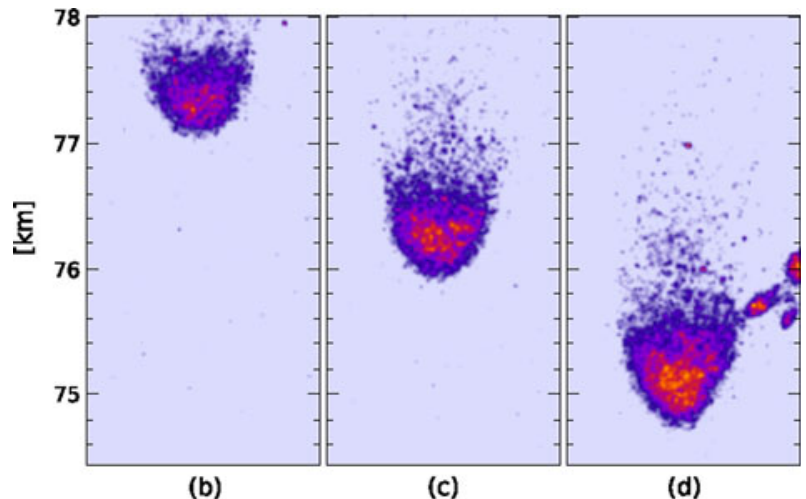

(b)

(c)

(d)

Reproduced from [Nudnova and Starlikovski, 2008]

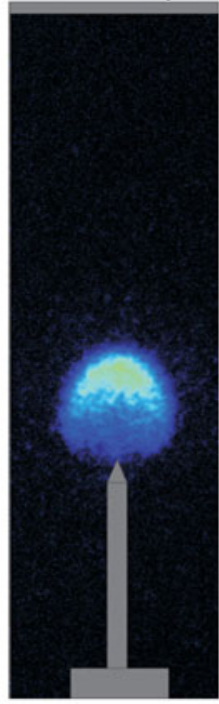

$5 \mathrm{~ns}$

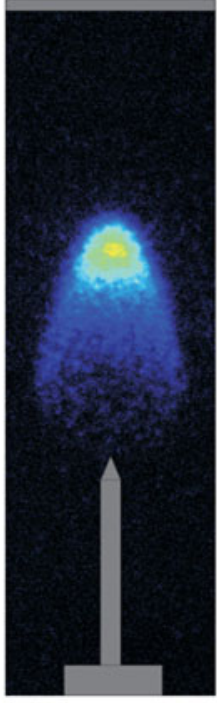

$10 \mathrm{~ns}$

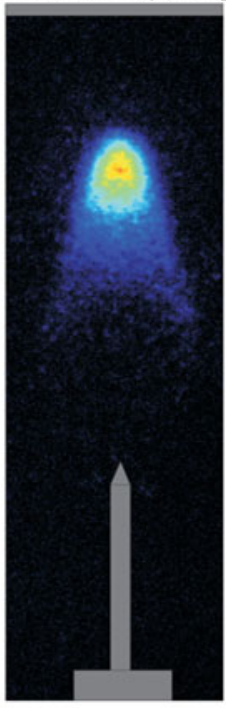

$15 \mathrm{~ns}$

$\Delta t=300 \mathrm{ps}, p=460$ Torr, $U=38 \mathrm{kV}$

Fig. 14 Top three successive images with a large sprite streamer recorded at 16,000 fps from Langmuir Laboratory, NM, on July 15, 2010 at 07:06:09 UT. The 500-m-wide streamer is the left streamer in Fig. 11. Bottom laboratory observations of a positive streamer in a $3 \mathrm{~cm}$ gap with an applied voltage of $38 \mathrm{kV}$. The images are $5 \mathrm{~ns}$ apart and the diameter of the streamer is 4-6 $\mathrm{mm}$. The observations were in air at a pressure of 460 Torr. The sprite and laboratory streamers show remarkable similar appearance. The laboratory images were reproduced from Nudnova and Starikovskii (2008) 
in importance with the exponentially increasing density at lower altitudes. Similarly, Liu and Pasko (2006) point out that quenching becomes more important at lower altitude and this may affect the scaling properties. Ebert et al. (2010) have discussed the general validity of the scaling laws and the conditions where corrections can and should be made. The processes in the streamer head ionization front are fast two-body processes for which the scaling laws should be valid, but in the streamer channel and in the glow region, the processes are slow and scaling may not be valid there. Briels et al. (2008) analyzed mainly laboratory observations, but also included sprite streamers observed by Gerken et al. (2000) and found the scaling appears to hold over 5 orders of magnitude in atmospheric density.

Modeling by Babaeva and Naidis (1997) and Liu and Pasko (2004) show that for a given ambient electric field, the streamer velocity and diameter increases linearly with streamer length. Hence, there should be a linear relationship between velocity and diameter (Naidis 2009), and this relationship should scale with atmospheric density. Based on the same model, the streamer brightness increases exponentially with time, as was verified by observations in Fig. 10 above. Recently, Naidis (2009) evaluated the velocity-diameter relationship for streamers incorporating laboratory observations by Briels et al. (2008) and Winands et al. (2008). The result is reproduced in Fig. 15 (left). Figure 15 (right) shows the result of a similar analysis of sprite streamers by Kanmae et al. (2012). The streamer diameters in both studies have been scaled to standard atmospheric sea level density. The laboratory streamers are both slower and smaller than what is found for the sprites, but both data sets appear to obey the same velocity-diameter relationship.

Note that, in Fig. 15, the electric field strength given is the peak electric field at the streamer head rather than the background ambient electric field used in Fig. 10. The field near the streamer head is substantially larger, by a factor of 3-5, due to the charges associated with the streamer. Models often assume spatial uniform conditions in a weakly ionized plasma (Morrill et al. 2002; Adachi et al. 2006; Kuo et al. 2005; Kanmae et al. $2010 \mathrm{~b}$ ), but this is not the case around the streamer head. Naidis (2009) points out that the maximum emission rates in streamers are not at the peak electric field, and Celestin and

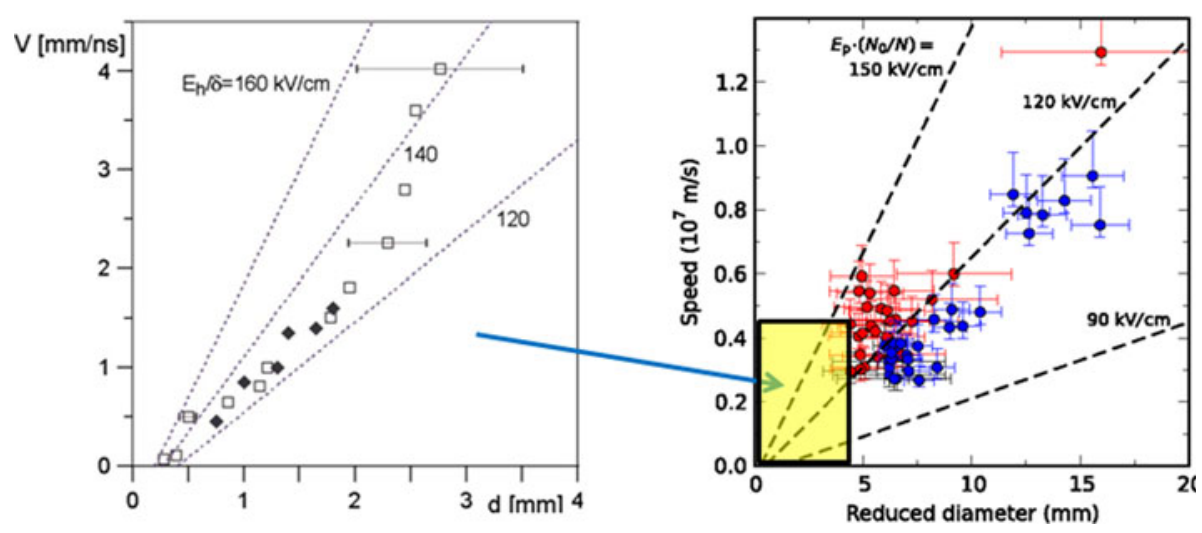

Fig. 15 Model streamer velocity versus diameter for different peak electric fields scaled to sea level density. The left panel, reproduced from Naidis (2009) Fig. 3, shows the relationship together with laboratory data from Briels et al. (2008) and Winands et al. (2008). The right panel, from Kanmae et al. (2012) Fig. 8, shows the same model relationship, but with sprite observations. The laboratory streamers are smaller and slower than sprite streamers, as indicated by the box mapping the Naidis plot surface onto the Kanmae plot, but both laboratory and sprite streamers appear to follow the same relationship 
Pasko (2010) find that, consequently, models assuming uniform spatial conditions may underestimate the peak electric field. Liu et al. (2006) evaluated the electric field and found the maximum electric field should be greater than $3 E_{\mathrm{k}}$ based on their model incorporating non-uniformities around the streamer head and satellite spectro-photometric data.

The streamer data set for the analysis by Kanmae et al. (2012), Fig. 15 right, is very limited. Although we have over the years recorded a large number of sprite streamers, the spatial resolution of most of these data is insufficient to measure streamer diameters with reasonable accuracy. But even with sufficient spatial resolution, only triangulated altitudes will provide sufficiently accurate scaling. We have few triangulated sprite positions as most observations have been made from one station only. To estimate altitude, it is then standard practice to assume the sprite to be collocated with or at the same range as the causal lightning discharge. The few published triangulated positions (Sentman et al. 1995; Lyons 1996; Wescott et al. 1998, 2001) indicate that the sprites may occur up to $100 \mathrm{~km}$ from the causal lightning. Thus, using the range to the strike to derive the altitude can introduce a serious error on the atmospheric density used to scale the observed streamer diameters. Our recent sprite observing campaigns have fielded several remote-observing sites to provide data for triangulation, and the observations used by Kanmae et al. (2012) have come from these campaigns. A study of the sprite locations relative to the lightning based on this newer data set has not yet been done.

It seems obvious to suggest that the differences in streamer diameter may be related to the scale of the discharges. The laboratory measurements were made in a $\sim 10-\mathrm{cm}$ chamber with the observations made close to the point of initiation, while the sprite observations are from streamers relatively much farther from the point of initiation. The modeling by Babaeva and Naidis (1997) and Liu and Pasko (2004) was done in a constant density environment. Recently Luque and Ebert (2010) investigated streamer propagation in air with varying density and found that, when the streamers move in varying density while conserving charge, the results are inconsistent with simple scaling. An example of their simulation was shown in Fig. 13 above where the streamer propagates from an altitude of 85 to $72 \mathrm{~km}$. Over this altitude range, the air density increases by a factor of 6 , but rather than a decrease in diameter, the streamer diameter actually grows from $\sim 300$ to $\sim 500 \mathrm{~m}$. Thus, for this simulation, 'simple' scaling with density does not appear to hold.

Streamer experiments have recently been performed in a 1-m air gap by Kochkin et al. (2012), and although they do not give any details on streamer width, they do report a typical width and velocity of $\sim 1 \mathrm{~cm}$ and $10^{6} \mathrm{~m} / \mathrm{s}$, respectively. This data point would fall well below the sprite data points in Fig. 15, right. For a width of $1 \mathrm{~cm}$, the measured sprite streamer widths data would suggest a velocity of $6 \times 10^{6} \mathrm{~m} / \mathrm{s}$ significantly faster than the $10^{6} \mathrm{~m} / \mathrm{s}$ given by Kochkin et al. (2012). Alternatively, the actual electric field near the streamer head could be lower, about $90 \mathrm{kV} / \mathrm{cm}$ or $\sim 3 E_{\mathrm{k}}$, than the fields inferred for the sprites which could also be the case (U. Ebert, personal communication, 2012). In any case, a general more extensive analysis of the streamer velocity/diameter/brightness relationship would be desirable.

\section{Future Research}

Sprite observations at high temporal resolution have provided new insights into sprite dynamics, and the observations have verified many details of model simulations. But they have also pointed out issues and problems to be investigated. A current example is the expectation of double-headed streamers (Qin et al. 2012) discussed in Sect. 3. These are not obvious in the high-speed images, and we are currently working on this issue. Another 
example is the relationship between streamer velocity and width discussed toward the end of Sect. 7. While Kanmae et al. (2012) found reasonable agreement between laboratory and sprite observations, streamer measurement by Kochkin et al. (2012) made in a 1-m air gap has a much different width/velocity ratio. Also, Luque and Ebert (2010) have pointed out that, in a varying density atmosphere, which obviously is the case for sprites, a 'simple' application of the streamer scaling laws may not hold.

Another line of investigations is the general relationship between sprites and the driving lightning electric field produced by tropospheric thunderstorm activity. More and more detailed 3D observations of currents and charge distributions in sprite-producing storms are being made providing for a rich data source for increasingly detailed modeling and comparison with optical sprite observations. For example, Li and Cummer (2011, 2012) have estimated electric charges in sprite streamers and have related streamer to the lightning electric field, which provide constraints on streamer propagation models; and Hager et al. (2012) compared electrical charge redistribution in the cloud tops to highspeed sprite imaging. In an earlier paper, Stenbaek-Nielsen et al. (2010), we noted that the relative occurrence rate of halo, $\mathrm{C}$-sprites, and carrots varies considerably between campaigns, and we speculated that this may be related to electric field parameters associated with the sprite-producing storm complexes.

Briels et al. (2008) and Nijdam et al. (2010) have investigated the minimal-size streamers present in laboratory experiments, and Ebert et al. (2010) present arguments for a minimum size in sprites as well. Briels et al. (2008) used the $\sim 20 \mathrm{~m}$ minimum widths of streamers observed by Gerken et al. (2000) as a possible minimum sprite streamer width, and the value, given the uncertainty, is in reasonable agreement with the laboratory observations (Briels et al. (2008), Fig. 5), indicating that scaling holds over 5 orders of magnitude in density. To make minimum width measurements in sprites will require, based on the data in Briels et al. (2008), a spatial resolution in the images below $10 \mathrm{~m}$. This can be attained using substantial longer focal length optics as found in 'off the shelf' astronomical telescopes, but this also decreases the field of view making the observations more tricky. However, a more serious problem will be signal to noise issues. The brightness of minimal streamers would likely be similar to the initial streamer simulated and compared to observations in Figs. 9 and 10 above (Liu et al. 2009a, b). This analysis indicates that we will need an improvement of about a factor of 100 in our ability to detect the streamer. Part of this could come from larger optics and the higher spatial resolution already required to resolve the streamers, but it is very doubtful that this would be enough. Thus, with the imagers currently available to us, it is not likely that we can observe minimum size sprite streamers.

An obvious topic for high-speed imaging is streamer splitting. To observe the temporal development of the process in details clearly will require higher framing rates than the 32,000 fps that we have used, or the 16,000 fps used to record the example in Fig. 11. Fortunately, sprite streamers are generally bright and well defined in the images. At 16,000 fps, they often saturate the imager. The bright streamer at center left in the top panel of Fig. 11 has a maximum pixel count of 10,079 out of a maximum of 16,383 (14 bit images). The smaller streamers at center right have counts in the 4,000-5,000 range. We have many good quality streamer images with significantly lower pixel counts. The event shown in Fig. 11 was recorded at $16,000 \mathrm{fps}$, but with $20 \mu$ s exposures. With the imager read out time being only $2 \mu \mathrm{s}$, we can increase the frame rate to almost 50,000 fps without any loss in image quality and we could even go to $100,000 \mathrm{fps}$ and still record good quality streamer images. We note that we have image quantity equal to that in Fig. 11 in our 32,000 fps data. Thus, there is easily enough light available to increase framing rate and spatial 
resolution substantially. However, our current camera digital technology will present a serious problem. This is imposed by the upper limit to the rate at which image data can be transferred to memory. Some increase in frame rate can be accommodated by a corresponding decrease in image size, but fewer pixels in each image would mean a smaller field of view. We could compensate for that by using a shorter-focal length lens to increase the field of view, but then, the spatial resolution would suffer. Another strategy would be to reduce image depth to 8 bits rather than 14. There are newer and more capable high-speed cameras available on the market today, but even they may be hard pressed to do these observations. However, imaging technology is steadily and rapidly improving.

Acknowledgments Discussions with Drs. U. Ebert, S. Cummer, N. Y. Liu, A. Luque, T. Neubert and V. Pasko are gratefully acknowledged. The data used in Fig. 7 were obtained on a sprite aircraft mission in July 2011 sponsored by the Japanese Broadcasting Corporation, NHK. Research funding has been provided by the US National Science Foundation grants to the University of Alaska Fairbanks, and the US Air Force Academy, and by DARPA through a grant to the University of Florida.

Open Access This article is distributed under the terms of the Creative Commons Attribution License which permits any use, distribution, and reproduction in any medium, provided the original author(s) and the source are credited.

\section{References}

Adachi T, Fukunishi H, Takahashi Y, Hiraki Y, Hsu RR, Su H-T, Chen AB, Mende SB, Frey HU, Lee LC (2006) Electric field transition between the diffuse and streamer regions of sprites estimated from ISUAL/array photometer measurements. Geophys Res Lett 33:L17803

Adachi T, Hiraki Y, Yamamoto K, Takahashi Y, Fukunishi H, Hsu RR, Su H-T, Chen AB, Mende SB, Frey HU, Lee LC (2008) Electric fields and electron energies in sprites and temporal evolutions of lightning charge moment. J Phys D Appl Phys 41:210-234. doi:10.1088/0022-3727/41/23/234010

Armstrong RA, Shorter JA, Taylor MJ, Suszcynsky DM, Lyons WA, Jeong LS (1998) Photometric measurements in the SPRITES '95 and '96 campaigns of nitrogen second positive $(399.8 \mathrm{~nm})$ and first negative (427.8 nm) emissions. J Atmos Sol Terr Phys 60:787-799

Armstrong RA, Suszcynsky DM, Lyons WA, Nelson TE (2000) Multi-color photometric measurements of ionization and energies in sprites. Geophys Res Lett 27:653-656. doi:10.1029/1999GL003672

Arrayas M, Ebert U, Hundsdorfer W (2002) Spontaneous branching of anode-directed streamers between planar electrodes. Phys Rev Lett 88:174502

Babaeva NY, Naidis GV (1997) Dynamics of positive and negative streamers in air in weak uniform electric fields. IEEE Trans Plasma Sci 25:375-379

Barrington-Leigh CP, Inan US, Stanley M (2001) Identification of sprites and elves with intensified video and broadband array photometry. J Geophys Res 106:1741-1750. doi:10.1029/2000JA000073

Bazelyan EM, Raizer Y (1998) Spark discharge. CRC Press, New York

Briels TMP, van Veldhuizen EM, Ebert U (2008) Positive streamers in air and nitrogen of varying density: experiments on similarity laws. J Phys D Appl Phys 41:234008. doi:10.1088/0022-3727/41/23/234008

Bucsela E, Morrill J, Heavner M, Siefring C, Berg S, Hampton D, Moudry D, Wescott E, Sentman D (2003) $\mathrm{N} 2(\mathrm{~B} 3 \mathrm{Pg})$ and $\mathrm{N} 2+(\mathrm{A} 2 \mathrm{Pu})$ vibrational distributions observed in sprites. J Atmos Sol Terr Phys 65:583-590. doi:10.1016/S1364-6826(02)00316-4

Celestin S, Pasko VP (2010) Effects of spatial non-uniformity of streamer discharges on spectroscopic diagnostics of peak electric fields in transient luminous events. Geophys Res Lett 37:L07-L804

Chamberlain JW (1995) Physics of the aurora and airglow. Classics in geophysics. American Geophysical Union, Washington, DC

Chanrion O, Neubert T (2008) A PIC-MCC code for simulations of long streamer propagation at sprite altitude. J Comput Phys 227:7222-7245. doi:10.1016/j.jcp.2008.04.016

Cummer SA, Füllekrug M (2001) Unusually intense continuing current in lightning causes delayed mesospheric breakdown. Geophys Res Lett 28:495-498. doi:10.1029/2000GL012214

Cummer SA, Jaugey N, Li J, Lyons WA, Nelson TE, Gerken EA (2006) Submillisecond imaging of sprite development and structure. Geophys Res Lett 33:L04104. doi:10.1029/2005GL024969 
Ebert U, Montijn C, Briels TMP, Hundsdorfer W, Meulenbroek B, Rocco A, van Veldhuizen EM (2006) The multiscale nature of streamers. Plasma Sources Sci Technol 15:S118-S129. doi:10.1088/ 0963-0252/15/2/S14

Ebert U, Nijdam S, Li C, Luque A, Briels T, van Veldhuizen E (2010) Review of recent results on streamer discharges and discussion of their relevance for sprites and lightning. J Geophys Res 115:A00E43. doi: 10.1029/2009JA014867

Fernsler RF, Rowland HL (1996) Models of lightning-produced sprites and elves. J Geophys Res 101:29653-29662

Franz RC, Nemzek J, Winckler JR (1990) Television of a large upward electrical discharge above a thunderstorm system. Science 249:48

Fukunishi H, Takahashi Y, Kubota M, Sakanoi K, Inan US, Lyons WA (1996) Elves: lightning-induced transient luminous events in the lower ionosphere. Geophys Res Lett 23:2157-2160

Gamerota WR, Cummer SA, Li J, Stenbaek-Nielsen HC, Haaland RK, McHarg MG (2011) Comparison of sprite initiation altitudes between observations and models. J Geophys Res 116:A02317. doi: 10.1029/2010JA016095

Gerken EA, Inan US (2002) A survey of streamer and diffuse glow dynamics observed in sprites using telescopic imagery. J Geophys Res 107(A11):13-44. doi:10.1029/2002JA009248

Gerken EA, Inan US (2003) Observations of decameter-scale morphologies in sprites. J Atmos Sol Terr Phys 65:567. doi:10.1016/S1364-6826(02)00333-4

Gerken EA, Inan US, Barrington-Leigh CP (2000) Telescopic imaging of sprites. Geophys Res Lett 27:2637-2640

Gordillo-Vázquez FJ (2008) Air plasma kinetics under the influence of sprites. J Phys D Appl Phys 41:234016. doi:10.1088/0022-3727/41/23/234016

Gordillo-Vázquez FJ (2010) Vibrational kinetics of air plasmas induced by sprites. J Geophys Res 115:A00E25. doi:10.1029/2009JA014688

Gordillo-Vázquez FJ, Luque A (2010) Electrical conductivity in sprite streamer channels. Geophys Res Lett 37:L16809. doi:10.1029/2010GL044349

Gordillo-Vázquez FJ, Luque A, Simek M (2012) Near infrared and ultraviolet spectra of TLEs. J Geophys Res 117:A05329. doi:10.1029/2012JA017516

Hager WW, Sonnenfeld RG, Feng W, Kanmae T, Stenbaek-Nielsen HC, McHarg MG, Haaland RK, Cummer SA, Lu G, Lapierre JL (2012) Charge rearrangement by sprites over a north Texas mesoscale convective system. J Geophys Res 117:D22101. doi:10.1029/2012JD018309

Hampton DL, Heavner MJ, Wescott EM, Sentman DD (1996) Optical spectral characteristics of sprites. Geophys Res Lett 23(1):89-92. doi:10.1029/95GL03587

$\mathrm{Hu}$ W, Cummer SA, Lyons WA (2007) Testing sprite initiation theory using lightning measurements and modeled electromagnetic fields. J Geophys Res 112:D13115. doi:10.1029/2006JD007939

Inan US, Barrington-Leigh C, Hansen S, Glukhov VS, Bell TF, Rairden R (1997) Rapid lateral expansion of optical luminosity in lightning-induced ionospheric flashes referred to as 'elves'. Geophys Res Lett 24:583-586

Kanmae T, Stenbaek-Nielsen HC, McHarg MG (2007) Altitude resolved sprite spectra with 3 ms temporal resolution. Geophys Res Lett 34:L07810. doi:10.1029/2006GL028608

Kanmae T, Stenbaek-Nielsen HC, McHarg MG, Haaland RK (2010a) Observation of blue sprite spectra at 10,000 fps. Geophys Res Lett 37:L13L808. doi:10.1029/2010GL043739

Kanmae T, Stenbaek-Nielsen HC, McHarg MG, Haaland RK (2010b) Observation of sprite streamer head's spectra at 10,000 fps. J Geophys Res 115:A00E48. doi:10.1029/2009JA014546

Kanmae T, Stenbaek-Nielsen HC, McHarg MG, Haaland RK (2012) Diameter-speed relation of sprite streamers. J Phys D Appl Phys 45:275203. doi:10.1088/0022-3727/45/27/275203

Kochkin PO, Nguyen CV, van Deursen APJ, Ebert U (2012) Experimental study of hard X-rays emitted from metre-scale positive discharges in air. J Phys D Appl Phys 45:202-425. doi:10.1088/00223727/45/42/425202

Kosar BC, Liu N, Rassoul HK (2012) Luminosity and propagation characteristics of sprite streamers initiated from small ionospheric disturbances at subbreakdown conditions. J Geophys Res 117:A08328. doi: 10.1029/2012JA017632

Kuo CL, Hsu RR, Chen AB, Su HT, Lee LC, Mende SB, Frey HU, Fukunishi H, Takahashi Y (2005) Electric fields and electron energies inferred from the ISUAL recorded sprites. Geophys Res Lett 32:L19103. doi:10.1029/2005GL023389

Kuo CL, Chen AB, Chou JK, Tsai LY, Hsu RR, Su HT, Frey HU, Mende SB, Takahashi Y, Lee LC (2008) Radiative emission and energy deposition in transient luminous events. J Phys D Appl Phys 41:234014. doi:10.1088/0022-3727/41/23/234014 
Li J, Cummer SA (2009) Measurement of sprite streamer acceleration and deceleration. Geophys Res Lett 36:L10812. doi:10.1029/2009GL037581

Li J, Cummer S (2011) Estimation of electric charge in sprites from optical and radio observations. J Geophys Res 116:A01301. doi:10.1029/2010JA015391

Li J, Cummer S (2012) Relationship between sprite streamer behavior and lightning-driven electric fields. J Geophys Res 117:A01317. doi:10.1029/2011JA016843

Li J, Cummer SA, Lyons WA, Nelson TE (2008) Coordinated analysis of delayed sprites with high-speed images and remote electromagnetic fields. J Geophys Res 113:D20206. doi:10.1029/2008JD010008

Liu NY (2010) Model of sprite luminous trail caused by increasing streamer current. Geophys Res Lett 37:L04102. doi:10.1029/2009GL042214

Liu N (2012) Multiple ion species fluid modeling of sprite halos and the role of electron detachment of O_in their dynamics. J Geophys Res 117:A03308. doi:10.1029/2011JA017062

Liu NY, Pasko VP (2004) Effects of photoionization on propagation and branching of positive and negative streamers in sprites. J Geophys Res 109:A04301. doi:10.1029/2003JA010064

Liu N, Pasko VP (2005) Molecular nitrogen LBH band system far-UV emissions of sprite streamers. Geophys Res Lett 32:L05104. doi:10.1029/2004GL022001

Liu N, Pasko VP (2006) Effects of photoionization on similarity properties of streamers at various pressures in air. J Phys D Appl Phys 39:327-334

Liu N, Pasko VP, Burkhardt DH, Frey HU, Mende SB, Su H-T, Chen AB, Hsu R-R, Lee L-C, Fukunishi H, Takahashi Y (2006) Comparison of results from sprite streamer modeling with spectrophotometric measurements by ISUAL instrument on FORMOSAT-2 satellite. Geophys Res Lett 33:L01101. doi: 10.1029/2005GL024243

Liu NY, Pasko VP, Adams K, Stenbaek-Nielsen HC, McHarg MG (2009a) Comparison of acceleration, expansion, and brightness of sprite streamers obtained from modeling and high-speed video observations. J Geophys Res 114:A00E03. doi:10.1029/2008JA013720

Liu N, Pasko VP, Frey HU, Mende SB, Su HT, Chen AB, Hsu RR, Lee LC (2009b) Assessment of sprite initiating electric fields and quenching altitude of a1Pg state of $\mathrm{N} 2$ using sprite streamer modeling and ISUAL spectrophotometric measurements. J Geophys Res 114:A00E02. doi:10.1029/2008JA013735

Liu N, Kosar B, Sadighi S, Dwyer JR, Rassoul HK (2012) Formation of streamer discharges from an isolated ionization column at subbreakdown conditions. Phys Rev Lett 109:025002. doi: 10.1103/PhysRevLett.109.025002

Luque A, Ebert U (2009) Emergence of sprite streamers from screening-ionization waves in the lower ionosphere. Nat Geosci 2(11):757-760. doi:10.1038/NGEO662

Luque A, Ebert U (2010) Sprites in varying air density: charge conservation, glowing negative trails and changing velocity. Geophys Res Lett 37:L06806. doi:10.1029/2009GL041982

Luque A, Ebert U (2011) Electron density fluctuations accelerate the branching of positive streamer discharges in air. Phys Rev E 84:046411. doi:10.1103/PhysRevE.84.046411

Luque A, Gordillo-Vázquez FJ (2011) Sprite beads originating from inhomogeneities in the mesospheric electron density. Geophys Res Lett 38:L04808. doi:10.1029/2010GL046403

Luque A, Gordillo-Vázquez FJ (2012) Mesospheric electric breakdown and delayed sprite ignition caused by electron detachment. Nat Geosci. doi:10.1038/NGEO1314

Lyons WA (1996) Sprite observations above the U.S. high plains in relation to their parent thunderstorm systems. J Geophys Res 101:29641-29652

Lyons WA, Armstrong RA, Bering EA, Williams ER (2000) The hundred year hunt for the sprite. EOS Trans Am Geophys Union 81:373-377

Marshall RA, Inan US (2005) High-speed telescopic imaging of sprites. Geophys Res Lett 32:L05L804. doi: 10.1029/2004GL021988

Marshall RA, Inan US (2006) High-speed measurements of smallscale features in sprites: sizes and lifetimes. Radio Sci 41:RS6S43. doi:10.1029/2005RS003353

McHarg MG, Haaland RK, Moudry D, Stenbaek-Nielsen HC (2002) Altitude-time development of sprites. J Geophys Res 107:1364. doi:10.1029/2001JA00028

McHarg MG, Stenbaek-Nielsen HC, Kammae T (2007) Observation of streamer formation in sprites. Geophys Res Lett 34:L06804. doi:10.1029/2006GL027854

McHarg MG, Stenbaek-Nielsen HC, Kanmae T, Haaland RK (2010) Streamer tip splitting in sprites. J Geophys Res 115:A00E53. doi:10.1029/2009JA014850

Mende SB, Rairden RL, Swenson GR, Lyons WA (1995) Sprite spectra; N2 1 PG band identification. Geophys Res Lett 22(19):2633-2636. doi:10.1029/95GL02827

Montanyà J, van der Velde $\mathrm{O}$, Romero D, March V, Solà G, Pineda N, Arrayas M, Trueba JL, Reglero V, Soula S (2010) High-speed intensified video recordings of sprites and elves over the western Mediterranean Sea during winter thunderstorms. J Geophys Res 115:A00E18. doi:10.1029/2009JA014508 
Morrill JS, Bucsela EJ, Pasko VP, Berg SL, Heavner MJ, Moudry DR, Benesch WM, Wescott EM, Sentman DD (1998) Time resolved N2 triplet state vibrational populations and emissions associated with red sprites. J Atmos Sol Terr Phys 60(7-9):811-829. doi:10.1016/S1364-6826(98)00031-5

Morrill J, Bucsela E, Siefring C, Heavner M, Berg S, Moudry D, Slinker S, Fernsler R, Wescott E, Sentman D, Osborne D (2002) Electron energy and electric field estimates in sprites derived from ionized and neutral N2 emissions. Geophys Res Lett 29:1462. doi:10.1029/2001GL014018

Moudry DR (2003) The dynamics and morphology of sprites. University of Alaska Fairbanks, Thesis

Moudry DR, Stenbaek-Nielsen HC, Sentman DD, Wescott EM (2003) Imaging of elves, halos and sprite initiation at $1 \mathrm{~ms}$ time resolution. J Atmos Solar Terr Phys 65:509-518. doi:10.1016/S1364-6826 (02)00323-1

Naidis GV (2009) Positive and negative streamers in air: velocity-diameter relation. Phys Rev E 79:057401

Neubert T, Rycroft M, Farges T, Blanc E, Chanrion O, Arnone E, Odzimek A, Arnold N, Enell C-F, Turunen E, Bosinger T, Mika A, Haldoupis C, van der Steiner RJ, Velde O, Soula S, Berg P, Boberg F, Thejll P, Christiansen B, Ignaccolo M, Fullekrug M, Verronen PT, Montanya J, Crosby N (2008) Recent results from studies of electric discharges in the mesosphere. Surv Geophys 29:71-137. doi: 10.1007/s10712-008-9043-1

Nijdam S, Moerman J, Briels T, van Veldhuizen E, Ebert U (2008) Stereo-photography of streamers in air. Appl Phys Lett 92:101502. doi:10.1063/1.2894195

Nijdam S, Geurts CGC, van Veldhuizen EM, Ebert U (2009) Reconnection and merging of positive streamers in air. J Phys D Appl Phys 42:045201

Nijdam S, van de Wetering FMJH, Blanc R, van Veldhuizen EM, Ebert U (2010) Probing photo-ionization: experiments on positive streamers in pure gases and mixtures. J Phys D Appl Phys 43:145204. doi: $10.1088 / 0022-3727 / 43 / 14 / 145204$

Nudnova MM, Starikovskii AY (2008) Streamer head structure: role of ionization and photoionization. J Phys D Appl Phys 41:234003. doi:10.1088/0022-3727/41/23/234003

Pasko VP (2007) Red sprite discharges in the atmosphere at high altitude: the molecular physics and the similarity with laboratory discharges. Plasma Sources Sci Technol 16:S13-S29. doi:10.1088/0963$0252 / 16 / 1 / \mathrm{S} 02$

Pasko VP (2010) Recent advances in theory of transient luminous events. J Geophys Res 115:A00E35. doi: 10.1029/2009JA014860

Pasko VP, Stenbaek-Nielsen HC (2002) Diffuse and streamer regions of sprites. Geophys Res Lett 29(10):1440. doi:10.1029/2001GL014241

Pasko VP, Inan US, Bell TF (1997) Sprites produced by quasielectrostatic heating and ionization in the lower ionosphere. J Geophys Res 102:4529-4561. doi:10.1029/96JA03528

Qin J, Celestin S, Pasko VP (2011) On the inception of streamers from sprite halo events produced by lightning discharges with positive and negative polarity. J Geophys Res 116:A06305. doi:10.1029/ 2010JA016366

Qin J, Celestin S, Pasko VP (2012) Formation of single and double-headed streamers in sprite-halo events. Geophys Res Lett 39:L05810. doi:10.1029/2012GL051088

Raizer YP (1991) Gas discharge physics. Springer, Berlin

Rycroft MJ, Odzimek A (2010) Effects of lightning and sprites on the ionospheric potential, and threshold effects on sprite initiation, obtained using an analog model of the global atmospheric electric circuit. J Geophys Res 115:A00E37. doi:10.1029/2009JA014758

Sentman DD, Wescott EM (1993) Video observations of upper atmospheric optical flashes recorded from an aircraft. Geophys Res Lett 20:2857

Sentman DD, Wescott EM, Osborne DL, Hampton DL, Heavner MJ (1995) Preliminary results from the Sprites94 aircraft campaign: 1. Red sprites. Geophys Res Lett 22:1205

Sentman DD, Wescott EM, Heavner MJ, Moudry DR (1996) Observations of sprite beads and balls. Abstract A71B-7, Suppl. To EOS, 77, F61

Sentman DD, Stenbaek-Nielsen HC, McHarg MG, Morrill J (2008) Plasma chemistry of sprite streamers. J Geophys Res 113:D11112. doi:10.1029/2007JD008941

Stanley M, Krehbiel P, Brook M, Moore C, Rison W, Abrahams B (1999) High speed video of initial sprite development. Geophys Res Lett 26:3201-3204. doi:10.1029/1999GL010673

Stenbaek-Nielsen HC, McHarg MG (2008) High time-resolution sprite imaging: observations and implications. J Phys D Appl Phys 41:234009.1-2340091.4. doi:10.1088/0022-3727/41/23/234009

Stenbaek-Nielsen HC, Moudry DR, Wescott EM, Sentman DD, Sabbas FA (2000) Sprites and possible mesospheric effects. Geophys Res Lett 27:3829-3832. doi:10.1029/2000GL003827

Stenbaek-Nielsen HC, McHarg MG, Kammae T, Sentman DD (2007) Observed emission rates in sprite streamer heads. Geophys Res Lett 34:L11105. doi:10.1029/2007GL029881 
Stenbaek-Nielsen HC, Haaland RK, McHarg MG, Hensley BA, Kanmae T (2010) Sprite initiation altitude measured by triangulation. J Geophys Res 115:A00E12. doi:10.1029/2009JA014543

Suszcynsky DM, Roussel-Dupré R, Lyons WA, Armstrong RA (1998) Blue-light imagery and photometry of sprites. J Atmos SolTerr Phys 60(7-9):801-809. doi:10.1016/S1364-6826(98)00027-3

Veronis G, Pasko VP, Inan US (1999) Characteristics of mesospheric optical emissions produced by lightning discharges. J Geophys Res 104(A6):12645-12656

Wescott EM, Sentman DD, Heavner MJ, Hampton DL, Lyons WA, Nelson T (1998) Observations of 'Columniform' sprites. J Atmos Terr Phys 60:733

Wescott EM, Stenbaek-Nielsen HC, Sentman DD, Heavner MJ, Moudry DR, Sao Sabbas FT (2001) Triangulation of sprites, associated halos and their possible relation to causative lightning and micrometeors. J Geophys Res 106:10467-10478

Winands GJJ, Liu Z, Pemen AJM, van Heesch EJM, Yan K (2008) Analysis of streamer properties in air as function of pulse and reactor parameters by iccd photography. J Phys D 41:234001. doi:10.1088/ 0022-3727/41/23/234001 